\title{
Variable stars in the open cluster NGC 6791 and its surrounding field ${ }^{\star}$
}

\author{
F. De Marchi ${ }^{1}$, E. Poretti ${ }^{2}$, M. Montalto ${ }^{1}$, G. Piotto ${ }^{1}$, S. Desidera ${ }^{3}$, L. R. Bedin ${ }^{4}$,

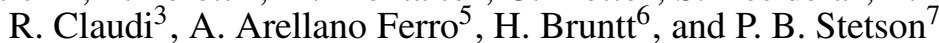

\author{
1 Dipartimento di Astronomia, Università di Padova, Vicolo dell'Osservatorio 2, 35122, Padova, Italy \\ e-mail: fabrizio.demarchi@unipd.it \\ 2 INAF - Osservatorio Astronomico di Brera, via E. Bianchi 46, 23807 Merate (LC), Italy \\ 3 INAF - Osservatorio Astronomico di Padova, Vicolo dell'Osservatorio 5, 35122, Padova, Italy \\ 4 Space Telescope Science Institute, 3700 San Martin Drive, Baltimore, MD 21218, USA \\ 5 Instituto de Astronomía, Universidad Nacional Autónoma de México, DF, Mexico \\ 6 School of Physics A28, University of Sydney, NSW 2006, Australia \\ 7 Herzberg Institute of Astrophysics, Victoria, Canada
}

Received 1 March 2007 / Accepted 12 June 2007

\section{ABSTRACT}

\begin{abstract}
Aims. This work presents a high-precision variability survey in the field of the old, super metal-rich open cluster NGC 6791.
Methods. The data sample consists of more than 75000 high-precision CCD time series measurements in the $V$ band obtained mainly at the Canada-France-Hawaii Telescope, with additional data from S. Pedro Mártir and Loiano observatories, over a time span of ten nights. The field covers an area of $42 \times 28 \mathrm{arcmin}^{2}$.

Results. We have discovered 260 new variables and re-determined periods and amplitudes of 70 known variable stars. By means of a photometric evaluation of the membership in NGC 6791, and a preliminary membership based on the proper motions, we give a full description of the variable content of the cluster and surrounding field in the range $16 \leqslant V<23.5$. Accurate periods can be given for the variables with $P \lessgtr 4.0 \mathrm{~d}$, while for ones with longer periods the limited time-baseline hampered precise determinations. We categorized the entire sample as follows: 6 pulsating, 3 irregular, 3 cataclysmic, 89 rotational variables and 61 eclipsing systems; moreover, we detected 168 candidate variables for which we cannot give a variability class since their periods are much longer than our time baseline.

Conclusions. On the basis of photometric considerations, and of the positions of the stars with respect to the center of the cluster, we inferred that 11 new variable stars are likely members of the cluster, for 22 stars the membership is doubtful and 137 are likely non-members. We also detected an outburst of about $3 \mathrm{mag}$ in the light curve of a very faint blue star belonging to the cluster and we suggest that this star could be a new U Gem (dwarf nova) cataclysmic variable.
\end{abstract}

Key words. stars: starspots - stars: statistics - stars: variables: general - stars: binaries: eclipsing - stars: novae, cataclysmic variables - open clusters and associations: individual: NGC 6791

\section{Introduction}

The photometric precision achieved by several ongoing transiting planet searches allows us to extend the census of variable stars down to very low amplitudes and faint magnitudes in selected sky regions. Variable stars are an important source of astrophysical information: from observations of them we are able to test several theories (e.g., evolutionary and pulsational models). Since all stars in an open cluster have essentially the same age, chemical composition and distance, the study of variables which are cluster members can put more severe constraints on the physical parameters. Comparisons can also be made between the variable stars of the cluster and those of the surrounding field.

In this paper, we present the study and the classification of 260 new variable stars that we found in the field of the open cluster NGC 6791, while for 70 already known variables we compare our results with the previous ones. NGC $6791(\alpha=$ $\left.19^{\mathrm{h}} 20^{\mathrm{m}} 53^{\mathrm{s}} ; \delta=+37^{\circ} 46^{\prime} 18^{\prime \prime}\right)$, is a rich and well studied open cluster. It is thought to be the one of the oldest and probably

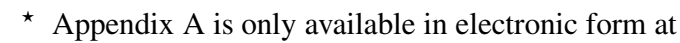
http://www. aanda.org the most metal-rich cluster known in our Galaxy. Its age is estimated to be about 8-9 Gyr (Carraro et al. 2006; King et al. 2005; Chaboyer et al. 1999; Stetson et al. 2003; Kaluzny \& Rucinski 1995); however, the white dwarf cooling sequence indicates a different value, i.e., 2.4 Gyr (Bedin et al. 2005). The most recent estimates of its metallicity are $[\mathrm{Fe} / \mathrm{H}]=+0.39$ (Carraro et al. 2006), $[\mathrm{Fe} / \mathrm{H}]=+0.47$ (Gratton et al. 2006), and $[\mathrm{Fe} / \mathrm{H}]=$ +0.45 (Anthony-Twarog et al. 2006). In this work we adopt for NGC 6791 a distance modulus $(m-M)_{V}=13.35 \pm 0.20$ mag and a reddening $E(B-V)=0.09$ mag (Carraro et al. 2006). The cluster is thus located at about $4.1 \mathrm{kpc}$ from the Sun.

Because of its extreme characteristics, NGC 6791 has been the target of many surveys (see Table 1 for a list of publications). Taking into account the fact that in four cases the same stars have two identification numbers $(\mathrm{V} 15 \equiv \mathrm{B} 7, \mathrm{~V} 56 \equiv \mathrm{V} 96, \mathrm{~V} 76 \equiv \mathrm{V} 85$ and $\mathrm{V} 77 \equiv \mathrm{V} 88$ ) and counting also the stars B4 and B8, the total number of known variable stars in the field of NGC 6791 to date was 123 (plus 7 suspected variables, proposed by Hartman et al. 2005).

In Sect. 2 below we describe our observations, in Sect. 3 we give details about the methods we employed in the search for 
Table 1. Previous variable star searches in NGC 6791.

\begin{tabular}{lrll}
\hline \hline Authors & Nr. of variables & IDs & Notes \\
\hline Kaluzny \& Rucinski (1993) (KR93) & 17 & V1-V17 & V15 B7 \\
Rucinski et al. (1996) (RK96) & 5 & V18-V21 and B8 & \\
Mochejska et al. (2002) (M02) & 47 & V22-V67 and B4 & B4 was previously catalogued by \\
& & & $\begin{array}{l}\text { Kaluzny \& Udalski (1992) as } \\
\text { a blue star, but not as variable. }\end{array}$ \\
Mochejska et al. (2003) (M03) & 7 & V68-V74 & \\
Kaluzny (2003) (K03) & 4 & V75-V78 & \\
Bruntt et al. (2003) (B03) & 19 & V79-V100 & V85 V76; V56 E96; V77 E88 \\
Mochejska et al. (2005) (M05) & 14 & V101-V114 & \\
Hartman et al. (2005) & 10 & V115-V124 & Plus 7 suspected variables \\
\hline
\end{tabular}

variable stars, which are themselves presented in Sect. 3.2. In Sect. 4 we describe the properties of the variable stars, focusing our attention on probable cluster members and some additional peculiar cases. The entire catalogue of variable stars is reported in an Appendix.

\section{Observations and data reduction}

We surveyed NGC 6791 to detect the transits of extrasolar planets (Montalto et al. 2007). The campaign covered 10 consecutive nights (from July 4, 2002 to July 13, 2002) and it was characterized by the continuous monitoring of the target on each clear night. Therefore, in addition to the planetary transit search, we could get access to the full variability content at $P \lessgtr 4.0 \mathrm{~d}$, both for the cluster and the surrounding field. Three telescopes were used:

1. the Canada-France-Hawaii Telescope (CFHT) in Hawaii equipped with the CFHT12k detector, composed of 12 CCDs of $4128 \times 2048$ pixels and covering a field of about $0.32 \mathrm{deg}^{2}$. Owing to the large number of bad columns, data from chip 6 could not be used, so we could get data over a $0.29 \mathrm{deg}^{2}$ field;

2. the San San Pedro Mártir (SPM) 2.1-m telescope equipped with the Thomson $2 \mathrm{k}$ detector and covering a field of about $6 \times 6 \operatorname{arcmin}^{2}$;

3. the Loiano 1.5-m telescope equipped with BFOSC + the EEV $1300 \times 1348$ B detector and covering a field of $11.5 \times$ $11.5 \operatorname{arcmin}^{2}$.

Table 2 gives details about the length of the observing nights while Fig. 1 shows the field of the CFHT survey and the edges of the Loiano and SPM surveys. The coordinates of the edges of our fields are also listed in Table 2. The field of the SPM observations is entirely included within chip 9 and the field of the Loiano observations partially covers chips 2, 3, 4, 8, 9 and 10 of CFHT (see Fig. 1). The luminosities of the new variables range from $V=23.2 \mathrm{mag}$ to $V \sim 16 \mathrm{mag}$ (near $1 \mathrm{mag}$ above the turnoff); brighter stars are saturated. The calibration of the CFHT, Loiano and SPM data have been performed by using the Kaluzny $\&$ Rucinski photometry (1995). More details on the data reduction procedure can be found in Montalto et al. (2007).

\section{The identification of variable stars}

The intensive monitoring of NGC 6791 allowed us to obtain tens of thousands of photometric time series for stars located in, close to, and far away from the cluster center. We have analysed 73331, 6055, and 2152 light curves obtained from the CFHT, Loiano and SPM telescopes respectively. The CFHT,
Table 2. The observation log for each night and the limits of the field of view at the 3 different observatories.

\begin{tabular}{|c|c|c|c|c|c|c|}
\hline \multirow[b]{2}{*}{ Night } & \multicolumn{2}{|c|}{$\overline{\text { Loiano }}$} & \multicolumn{2}{|c|}{$\overline{\text { SPM }}$} & \multicolumn{2}{|c|}{$\overline{\mathrm{CFHT}}$} \\
\hline & $\begin{array}{c}t_{\text {start }} \\
\text { (HJD- }\end{array}$ & $\begin{array}{c}t_{\text {end }} \\
52400)\end{array}$ & \multicolumn{2}{|c|}{ (HJD-2 452 400) } & \multicolumn{2}{|c|}{ 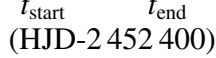 } \\
\hline $1 \mathrm{st}$ & & & & & 59.82 & 59.96 \\
\hline 2nd & & & & & 60.83 & 61.02 \\
\hline $3 \mathrm{rd}$ & 62.48 & 62.63 & 61.68 & 61.95 & 61.94 & 62.07 \\
\hline 4th & 63.41 & 63.63 & 62.68 & 62.96 & 62.76 & 63.07 \\
\hline 5 th & 64.38 & 64.64 & 63.68 & 63.96 & 63.88 & 64.10 \\
\hline 6 th & 65.39 & 65.62 & 64.69 & 64.98 & 64.77 & 65.11 \\
\hline 7 th & & & 65.70 & 65.82 & & \\
\hline 8th & & & 66.69 & 66.97 & & \\
\hline 9th & & & 67.67 & 67.98 & 67.77 & 68.10 \\
\hline 10th & & & 68.69 & 68.87 & 68.76 & 69.11 \\
\hline$\alpha_{\min }$ & $19^{\mathrm{h}} 20^{\prime}$ & & $19^{\mathrm{h}} 20$ & 6.3 & $19^{\mathrm{h}} 19$ & \\
\hline$\alpha_{\max }$ & $19^{\mathrm{h}} 21^{1}$ & & $19^{\mathrm{h}} 21^{1}$ & & $19^{\mathrm{h}} 22$ & \\
\hline$\delta_{\min }$ & $37^{\circ} 41^{\prime}$ & & $37^{\circ} 43$ & & $37^{\circ} 36$ & \\
\hline$\delta_{\max }$ & $37^{\circ} 53$ & & $37^{\circ} 50$ & & $38^{\circ} 4^{\prime} 2$ & \\
\hline
\end{tabular}

Loiano and SPM time series are composed of about 250, 60 and 170 datapoints, respectively. The observations, intended to detect photometric transits, were performed in the $V$ band only.

\subsection{The search for variable candidates}

To search for variable stars, we calculated the best "sinusoid plus constant" fit for all light curves (Vanicek 1971; Ferraz-Mello 1981). We evaluated the goodness of the fit by calculating parameters related to the reduction of the initial variance obtained by introducing the periodic term. These parameters are the reduction factor (Vanicek 1971) and the coefficient of spectral correlation $S(v)$ (Ferraz-Mello 1981).

Owing to the huge number of light curves, we need one or more parameters to discover the variability. Toward this goal, we considered the parameter $r$ defined as $r=\log _{10} S_{\text {max }}$, where $S_{\text {max }}$ is the maximum value of $S$ (i.e., the one corresponding to the frequency of the best-fit sinusoid in the Ferraz-Mello method). If a star does not show variability the introduction of a sinusoid does not improve the fit and then $S_{\max }$ is close to zero (no variance reduction) and $r \ll 0$; on the other hand, a sine-shaped variability strongly reduces the variance ( $S$ close to 1 ) and hence $r=0$. The purpose was to use the $r$ parameter as a tracer of variability for short-period (i.e., intranight) variability.

To search for long-period variability, we introduced a second parameter, more sensitive to the night-to-night variations. We calculated the mean magnitude $V_{i}$ and the standard 


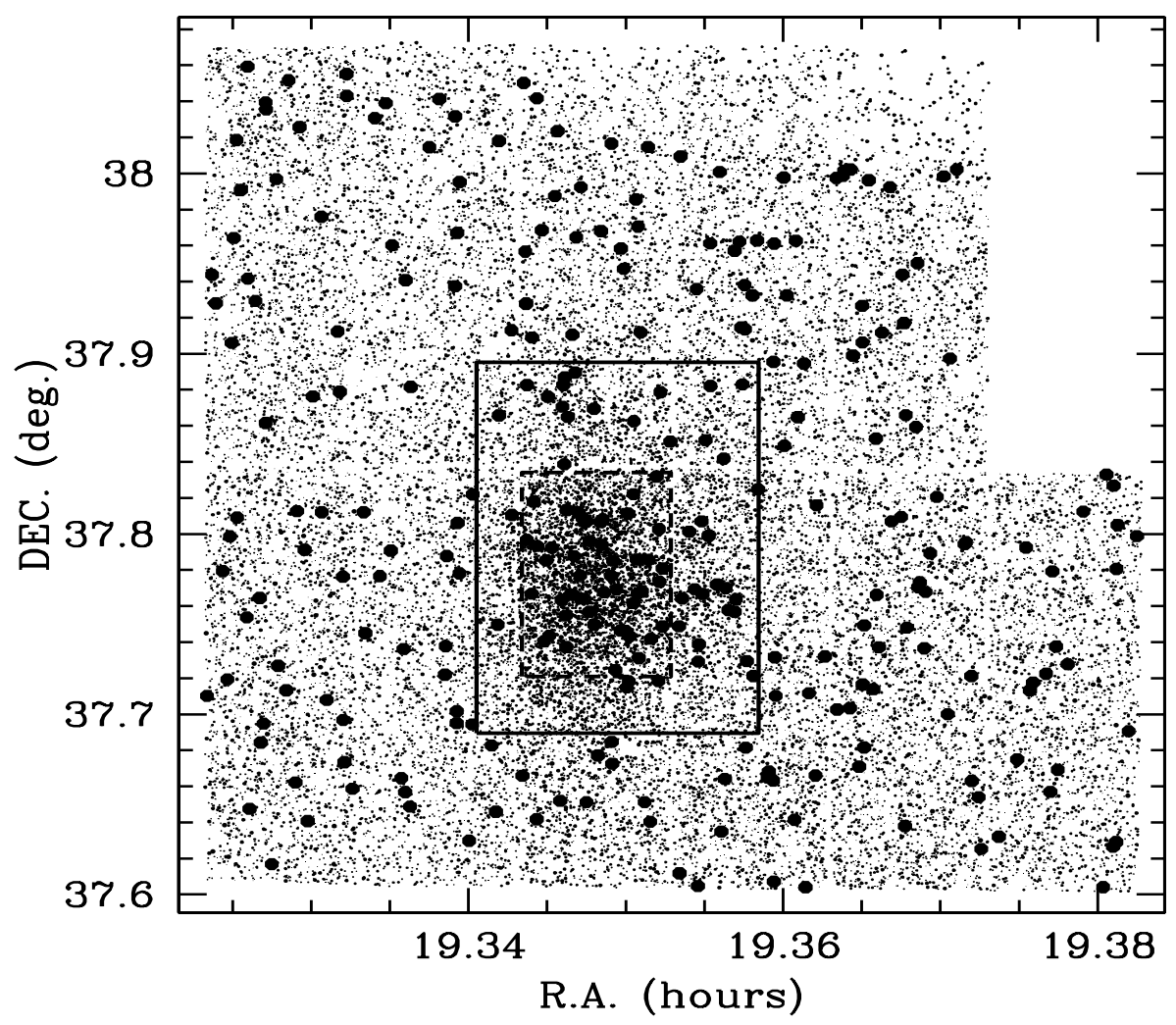

Fig. 1. Field of view $\left(42 \times 28 \mathrm{arcmin}^{2}\right)$ of the CFHT image. The chips are numbered in increasing order from left to right from chip 1 (top left) to chip 12 (bottom right); stars of chip 6 are not plotted since we found it impossible to derive accurate photometry. Dashed and solid lines are the edges of the SPM and Loiano fields, respectively. Variable stars are also plotted: blue dots are pulsating variables, purple dots are irregular and cataclysmic variables, green dots and cyan dots are eclipsing systems (EA/EB-type and EW-type, respectively). Finally, red and yellow dots are rotational and long-period variables, respectively. deviation $\sigma_{i}$ on each night, and after that we calculated the parameter $s$ defined as:

$s=\log _{10} \frac{\Delta V}{\bar{\sigma}}$

where $\Delta V$ is the peak-to-peak difference and $\bar{\sigma}$ is the mean of the $\sigma_{i}$ over all nights.

To test the capability of the $r$ and $s$ parameters to detect variable stars, we prepared a sample containing two types of light curves: 7722 artificial constant light curves (see Montalto et al. 2007, for details) and 70 light curves of already known variable stars which are included in our CFHT field. In Fig. 2 we plot $r$ vs. $s$ for the light curves of constant stars (small points) and of variable stars (large points). The variable stars are substantially apart from the constant stars and most have $r \gtrsim-1$. The variable stars with $r \lesssim-1$ and superposed on constant stars are mostly EA-type stars or irregular stars (e.g., cataclysmic variables). Among variables (i.e., large dots in Fig. 2), the stars with small $s$ have short periods $(P \leq 0.50 \mathrm{~d})$, while stars with large $s$ have long periods. Therefore, we can conclude that the combination of the $r$ and $s$ parameters is a good tracer of variability.

To detect the variable stars in our sample of $\sim 82000$ light curves we first selected in an automatic way all the stars with $r \geq-2.0$, according to the test described above. We thereby reduced the huge initial sample to $\sim 6500$ stars. After the calculation of the amplitude spectrum of their time series, we adopted as a second selection criterion a signal-to-noise ratio $(S / N)$ greater than 4.0 around the highest peak in the amplitude spectrum. This procedure allowed us to reduce our sample to $\sim 900$ stars, i.e., $1.1 \%$ of the whole initial sample. Further checks have been made by examining the light curves of a random sample of stars with $r<-2.0$, large $s$ and $3.5<S / N<4.0$, but we did not find any additional variables.

Our approach allowed us to detected hundreds of stars showing peaks in their power spectra at $f=1.00 \mathrm{~cd}^{-1}$, at

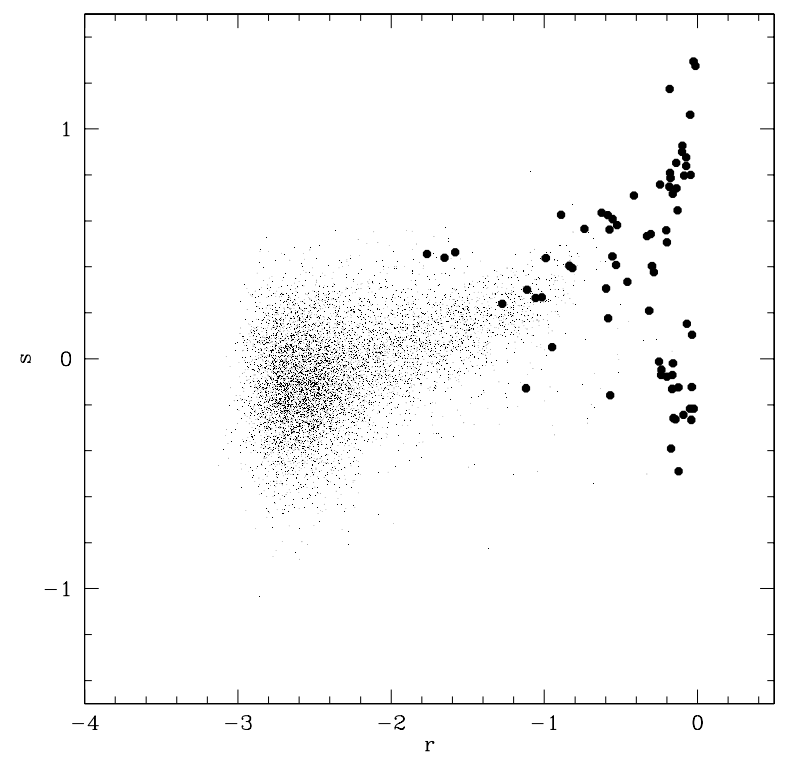

Fig. 2. Grey points: $r$ parameter vs. $s$ parameter for constant light curves. Black dots: parameters derived from our light curves for the variable stars previously detected in our field.

$f \leq 0.05 \mathrm{~cd}^{-1}$, or at $f=0.6 \mathrm{~cd}^{-1}$. The first two spurious periodicities are common and can be ascribed to small misalignments in the mean magnitudes from one night to the next or recurrent drifts (caused by small color effects, for example) in the intranight light curves. We suggest that the latter one is probably a photometric artefact occurring in some particular cases of blended stars, or stars close to CCD edges, or bad pixels. They have been considered as not reliable enough to infer a physical light variability. In our opinion, only the combination of automatic procedures and visual inspection allowed us to identify the three classes $(P=1.00 \mathrm{~d}, P=1.6 \mathrm{~d}, P \gg 10 \mathrm{~d})$ of spurious 
variables in the huge number of $\sim 82000$ light curves. In particular, we note that the identification of the whole sample of eclipsing binaries has been confirmed by the application of the box fitting technique (BLS, Kòvacs et al. 2002), used by Montalto et al. (2007) to detect planetary transits.

At the end of the variable star identification, we were left with 330 cases to be characterized. Since we rejected about 2/3 of the sample selected by means of the $r, s$ parameters, we are confident we have not applied overly strict constraints in the candidate selection.

\subsection{The cross check with previous surveys of NGC 6791}

When comparing our field of view with those of other surveys, we found that 81 known variable stars are included. The CFHT survey failed to detect 45 known variable stars: seventeen stars (V22, V24, V26, V28, V30, V35, V36, V47, V50, V57, V61, V63, V64, V102, V103, V104, V105) are outside the CFHT field of view; 4 stars (V71, V106, V113 and V120) lie between two chips; 23 stars (V1, V6, V13, V19, V33, V39, V45, V49, $\mathrm{V} 54, \mathrm{~V} 56 \equiv \mathrm{V} 96, \mathrm{~V} 65, \mathrm{~V} 66, \mathrm{~V} 67, \mathrm{~V} 69$, V70, V72, V73, V74, $\mathrm{V} 77 \equiv \mathrm{V} 88, \mathrm{~V} 78, \mathrm{~V} 81$, V97 and V112) are saturated; and the V76 data are useless.

Among the 81 known variable stars that we have observed, not all of them display variability in our sample: 4 stars (V10, V18, V21, V32) are previously classified as long-period detached eclipsing variables and we did not observed eclipses. We are not able to confirm the period of 15.24 days for V68 (M03), likely because of our shorter time baseline and the small amplitude of this variable (about $0.003 \mathrm{mag}$ in $V$-band, M03). Finally, we cannot confirm the variability of six stars (V20, V79, V84, V98, V99, V116) and of the seven suspected variables found by $\mathrm{H} 05$, since our data do not show any trace of variability.

Among the sample of the stars missing from the CFHT field, we identified 22 stars in the Loiano and SPM data sets (V6, V13, V19, V20, V33, V45, V54, V56 $\equiv$ V96, V65, V66, V67, $\mathrm{V} 70, \mathrm{~V} 71, \mathrm{~V} 73, \mathrm{~V} 74, \mathrm{~V} 76 \equiv \mathrm{V} 85, \mathrm{~V} 77, \mathrm{~V} 78, \mathrm{~V} 81, \mathrm{~V} 97, \mathrm{~V} 106$ and V113). However, owing to the smaller signal-to-noise ratio $(S / N)$, the small number of datapoints and (in the case of the Loiano data) the limited survey time, we could only confirm the variability of stars V56 $\equiv \mathrm{V} 96$, V66 and V76 $\equiv \mathrm{V} 85$.

Throughout this paper we use the existing names for the already known variables; to identify the new ones discovered in our survey we used the five-digit number assigned by the DAOPHOT package followed by the number of the chip which the star belongs to. Accurate astrometry is provided to identify the stars on the sky. Moreover, all light curves of the variables will be available on CDS.

\section{The variable star content of NGC 6791 and its surrounding field}

The CFHT measurements are quite precise, thus the light curves are generally very well defined for $P<4 \mathrm{~d}$. On the other hand, the periods and the shapes are uncertain for $P>4 \mathrm{~d}$, since the observations only covered 2.5 cycles or less. We refer to Montalto et al. (2007) for a full description of the photometric errors. In order to evaluate the precision in the study of the variable stars, we calculated the standard deviations of the Fourier leastsquares fits (truncated at the last significant term for the given star) for the 138 light curves having very good phase coverage. The precision was found to be better than $0.010 \mathrm{mag}$ in 73 cases $(53 \%)$, and better than $0.020 \mathrm{mag}$ in a total of 122 cases $(88 \%)$,

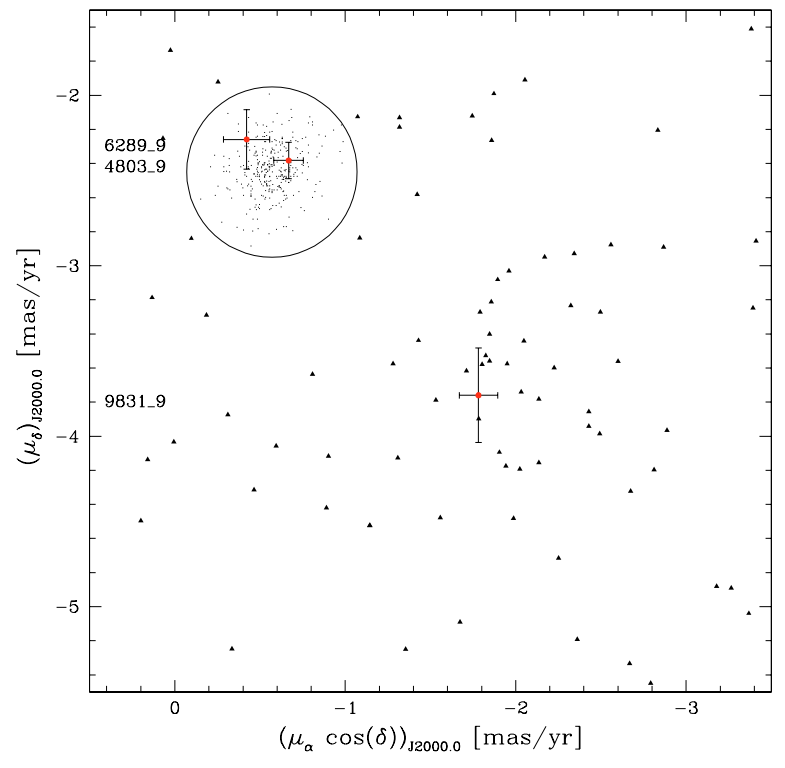

Fig. 3. Proper-motion vector-point diagram for the inner region of NGC 6791 (from Bedin et al. 2006). The circle (centered on the absolute proper motion of the cluster) represents a safe limit corresponding to $0.5 \mathrm{mas} / \mathrm{yr}$. Triangles represent non-members, points with error bars are the new variables 06289_9, 04803_9 and 09831_9.

as expected for stars ranging from $V \sim 16.0$ to $V \sim 22.5$. The discussion based is mostly on the CFHT data, which are by far the most numerous, precise and homogeneous; however, for some variables we have used data from Loiano and SPM in a very profitable way. As an example, only the longitude spread of the three observatories allowed us to derive the periods of the eclipsing binaries 00645_10, V107, V12, V109 and of the rotational variable 03079_9.

To proceed in the definition of the variable star content of NGC 6791 and its surrounding field, we calculated the power spectra of the data for all the 330 candidate variables by using the least-squares iterative sine-wave search (Vanicek 1971) and the Phase Dispersion Minimization (Stellingwerf 1978) methods. Differences have been examined and resolved. The separation into different classes of variable stars has been made on the basis of the light curve parameters (period, amplitude, Fourier coefficients) and standard photometric values $(V, B-V$, $V-I)$, when available. The period estimates have been refined by means of a least-squares procedure (MTRAP, Carpino 1987) and appropriate error bars have also been calculated. At the end of the process we get six pulsating stars with $P<0.6 \mathrm{~d}$, three irregular variables, three cataclysmic variables (CVs), 31 detached or semi-detached eclipsing binaries, 29 contact binaries, 90 rotational variables, 167 stars showing clear night-to-night variability on timescales too long for periods to be determined over our 9.2-d baseline. We adopt preliminary membership probabilities based on proper motion measurements kindly provided to us by K. Cudworth (private communication) for 35 stars. Moreover, for three new variable stars we adopted membership probabilities based on proper motions performed by Bedin et al. (2006) (hereafter B06, see Fig. 3).

For the other stars, we consider their position in color-magnitude diagrams (CMDs), and their distance from the center of the cluster to infer whether they belong to the cluster (for EW-type stars we also utilize the $P-L-C$ relation of Rucinski (2003). Toward this end, we plotted the radial distribution of all stars in Fig. 4. We see that at a distance of $\sim 10^{\prime}$ from the center 
Table 3. Pulsating, irregular and cataclysmic variables. $V$ is the minimum brightness for CVs and irregular, the mean brightness for pulsating variables. $T_{0}$ is the time of maximum brightness for pulsating stars. Hereafter, the labels "k" and "s" indicate that the $B-V$ color index is taken from Kaluzny \& Rucinski (1995) or Stetson et al. (2003), respectively.

\begin{tabular}{|c|c|c|c|c|c|c|c|c|c|c|}
\hline Star & Type & $\alpha_{2000}$ & $\delta_{2000}$ & $\begin{array}{c}V \\
{[\mathrm{mag}]}\end{array}$ & $\begin{array}{c}\langle B-V\rangle \\
{[\mathrm{mag}]}\end{array}$ & $\begin{array}{c}\langle V-I\rangle \\
{[\mathrm{mag}]}\end{array}$ & Ref. & $\begin{array}{l}T_{0} \\
\text { [HJD-2 452 400] }\end{array}$ & $\begin{array}{l}\text { Period } \\
\text { [d] }\end{array}$ & $\begin{array}{l}\text { Ampl. } \\
{[\mathrm{mag}]}\end{array}$ \\
\hline \multicolumn{11}{|c|}{ Pulsating variables } \\
\hline V123 & HADS & 19.362064 & 37.666034 & 17.08 & 0.45 & & $\mathrm{k}$ & 59.559 & 0.06026 & 0.14 \\
\hline $01497 \_12$ & HADS & 19.379083 & 37.812419 & 16.06 & & & & 59.528 & 0.07227 & 0.40 \\
\hline 00311_7 & SXPhe & 19.324628 & 37.716768 & 23.17 & & & & 59.605 & 0.10443 & 0.10 \\
\hline 00224_10 & SXPhe & 19.353639 & 37.710163 & 21.72 & 0.71 & 1.06 & $\mathrm{~s}$ & 59.801 & 0.12261 & 0.20 \\
\hline 03653_3 & $\mathrm{RRc}$ & 19.347147 & 37.992413 & 17.21 & 0.57 & 0.58 & $\mathrm{k}$ & 59.937 & 0.32654 & 0.39 \\
\hline 00345_1 & $\mathrm{RRab}$ & 19.325082 & 37.964170 & 18.28 & & & & 60.151 & 0.57866 & 0.72 \\
\hline \multicolumn{11}{|c|}{ Irregular variables } \\
\hline V92 & IRR & 19.350754 & 37.766876 & 18.10 & 0.91 & & $\mathrm{k}$ & & & 0.10 \\
\hline V83 & IRR & 19.346220 & 37.737232 & 19.10 & 1.02 & 1.05 & $\mathrm{k}$ & & & 0.07 \\
\hline V93 & IRR & 19.351452 & 37.785687 & 18.12 & 0.98 & 1.03 & $\mathrm{~s}$ & & & 0.04 \\
\hline \multicolumn{11}{|c|}{ Cataclysmic variables } \\
\hline $\mathrm{V} 15(=\mathrm{B} 7)$ & $\mathrm{CV}$ & 19.352057 & 37.799019 & 18.26 & 0.20 & & $\mathrm{k}$ & & & 0.06 \\
\hline B8 & $\mathrm{CV}$ & 19.343262 & 37.747833 & 20.64 & -0.23 & 0.78 & $\mathrm{k}$ & & & 2.27 \\
\hline 06289_9 & CV (?) & 19.348976 & 37.770355 & 22.80 & 0.25 & 0.88 & $\mathrm{~s}$ & & & 3.10 \\
\hline
\end{tabular}

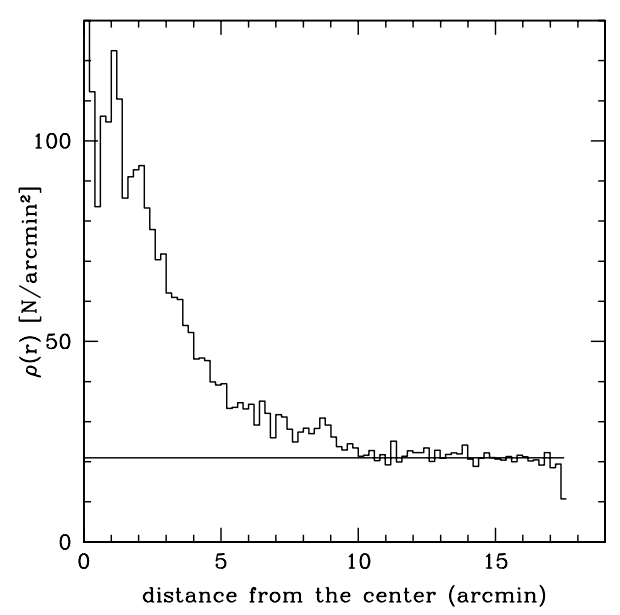

Fig. 4. Stellar density $\rho$ (number of stars per square arcminute) as a function of the distance from the center. The straight line represents the mean stellar density at distances greater than $10^{\prime}$.

of the cluster, the stellar density becomes near constant (about $21 \mathrm{star} / \operatorname{arcmin}^{2}$ ). Thus, we adopt the value of $10^{\prime}$ as the external limit of the cluster and we consider "likely non-members" the variables located farther from the cluster center.

\subsection{Pulsating variables}

The main characteristics of our variables are listed in Table 3 and their light curves are shown in Fig. 5. The classification as High-Amplitude Delta Sct (HADS), SX Phe, RRc or RRab stars is based on the parameters of the Fourier decomposition (Poretti 2001). In all cases, the $\phi_{21}$ Fourier parameters are on the progressions described by the different classes. We note that our period for V123 is quite different from that given by H05 (0.107 d). Error bars on the periods are in the range $1-6 \times 10^{-5} \mathrm{~d}$.

Both RR Lyr variables are too faint to belong to NGC 6791. Since they have $V=17.21$ (03653_3) and $V=18.28$ (00345_1), their distance moduli greatly exceed that of the cluster.
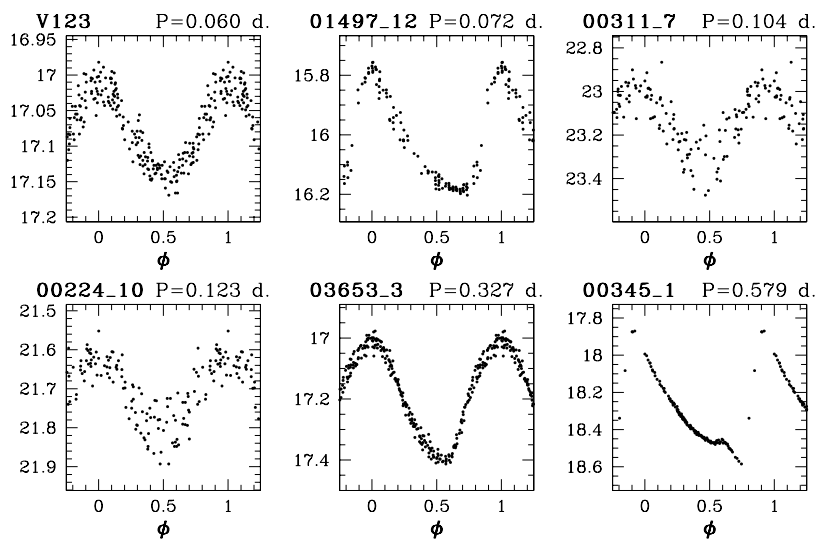

Fig. 5. Light curves of pulsating variables. V123 and 01497_12 are probably HADS stars, 00311_7 and 00224_10 are SX Phe stars, 03653_3 and 00345_1 are RR Lyr stars.

This is also true for the very faint and short-period stars 00311_7 $(V=23.17)$ and 00224_10 $(V=21.72)$; therefore, it is more likely that they are Pop. II stars. On the other hand, using the $P-L$ relation given by McNamara (2000), we get distance moduli of 14.50 and 13.78, respectively, for V123 and 01497_12. These distance moduli and the distance from the cluster center (12' and $22^{\prime}$, respectively) suggest that they do not belong to the cluster, though they are not very far from it. Therefore, they are probably Pop. I stars and hence High Amplitude $\delta$ Scuti stars.

Moreover, there are several variables whose light curves are very similar to those of Cepheid variables; the Fourier decomposition of some light curves (in particular the large amplitude ones, i.e., 00913_5, 01659_8, V46 and 01431_10, but also 01606_11,02285_10,00122_4 and 03056_3) yields parameters typical for Cepheid light curves. However, most of these variables are quite faint and the Period-Luminosity relation for Cepheids (Tammann et al. 2003) yields distances in the range $39-171 \mathrm{Kpc}$. It is difficult to say whether these stars are nearby rotational variables (see below) in the Milky Way or very 
distant pulsating variables. For our present purposes, these stars have been included among the rotational variables listed in the Appendix.

The puzzling nature of all these apparently distant stars (i.e., the Cepheid-like ones, the RR Lyr and the faint SX Phe variables discussed above) deserves further investigation by means of spectroscopic and/or kinematic data.

\subsection{Irregular variables}

Table 3 also lists three irregular variables: these stars lie on the middle Main Sequence and are all located less than $3^{\prime}$ from the cluster center; thus we suggest that they belong to the cluster. V92 and V83 were previously defined as "periodic variables" by B03. Indeed, we noticed fast variability in our light curves (Fig. 6), but, more noticeably, the mean magnitude is also changing from night to night. The long periods given by M03 are not able to explain either the short- or the longer-timescale variability; actually, we could not detect any periodic term. We also detected no trace of periodicity in V93 (Fig. 6); we suspect that the periods given by M05 and B03 are spurious, since they are close to $1.0 \mathrm{~d}$ ( 0.99 and $0.94 \mathrm{~d}$, respectively) and they could be produced by the irregular fluctuations.

We can conjecture that these variables are eruptive variables observed in a quiescent phase, in which rapid and/or slow changes with smaller amplitude can be observed; they resemble the case of V15 (see Sect. 4.3). We have no reliable indications about the membership probabilities.

\subsection{Cataclysmic variables}

As regards V15: M03 and M05 detected variability over the range of 3 mag and observed outbursts of about 0.5-1.0 mag; from our side, we could see a 0.15 -mag variability in our light curves (Fig. 6), corresponding to the quiescent phase. V15 is very probably a NGC 6971 member, since the Cudworth propermotion membership probability is very high (98\%).

Both the position of the faint blue star 06289_9 in the two-colour diagram and the shape of its light curve (Fig. 7) strongly suggest that this star could be a new cataclysmic variable (U Gem-type, dwarf nova). Moreover, we know that this object is a cluster member (see Fig. 3). The star shows an outburst of about 3 mag and, though we did not observe the entire brightening, we would highlight that the magnitude was still increasing on the first night; thus we are able to say that the maximum brightness was reached immediately after.

We can estimate the orbital period, $P_{\text {orb}}$, and the recurrence time, $T_{n}$, from the decay time, $\tau_{\mathrm{d}}=\Delta t / \Delta m$ [days $\mathrm{mag}^{-1}$ ] and the amplitude, $\Delta m$ (Warner, 1995, Eqs. (3.5), (3.1), respectively). Assuming for $\Delta t$ and $\Delta m$ the values $3.33 \pm 0.50 \mathrm{~d}$ and $2.87 \pm$ $0.31 \mathrm{mag}$ respectively, we find $P_{\text {orb }}=2.54 \pm 1.41 \mathrm{~h}$ and $T_{n}=$ $13.9 \pm 10.6$ d. However, our light curve (Fig. 7) seems to rule out $T_{n}$ values shorter than $8 \mathrm{~d}$.

The variable B8 shows a large-amplitude light curve (Fig. 7) over a quite short $7 \mathrm{~d}$ time span. The cataclysmic nature of B8 has been confirmed spectroscopically by Kaluzny et al. (1997) who also notes that B8 exhibits red $V-I$ colour while in a low state.

Following the same procedure used for 06289_9 and assuming $\tau_{\mathrm{d}}=1.3 \pm 0.3 \mathrm{~d} \mathrm{mag}^{-1}$ for B8, we find $P_{\text {orb }}=2.97 \pm 1.63 \mathrm{~h}$ and $T_{n}=11.4 \pm 8.5 \mathrm{~d}$. The $T_{n}$ value is compatible with the $7 \mathrm{~d}$ periodicity (Fig. 7). A membership probability is not available for B8. However, using the Eqs. (3.3) and (3.4) after

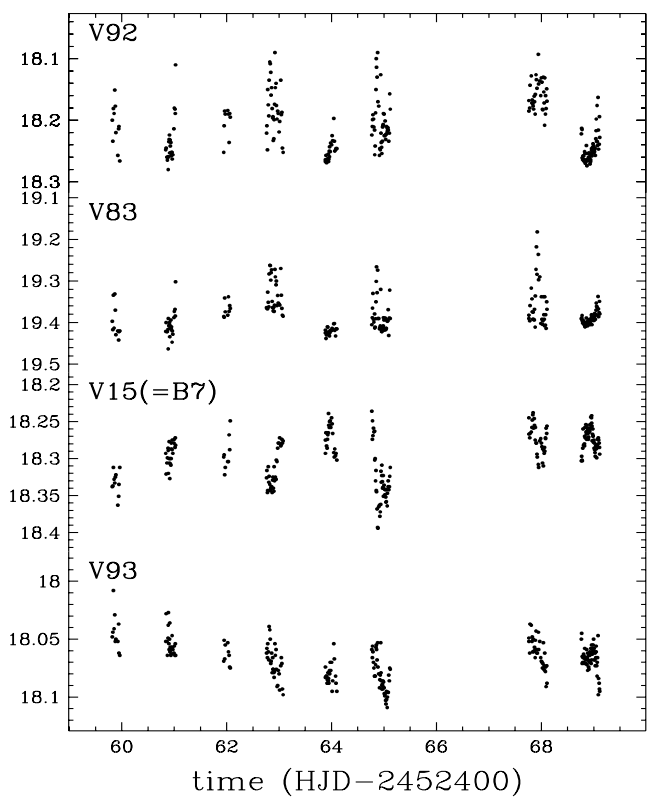

Fig. 6. Variable stars showing irregular fluctuations.

Warner (1995), we obtain $M_{V \min }=8.06 \pm 0.68 \mathrm{mag}$ and $M_{V \max }=$ $4.97 \pm 0.42 \mathrm{mag}$. In turn, these values give two estimates for the distance modulus of B8, i.e., $13.82 \pm 0.68 \mathrm{mag}$ and $14.20 \pm 0.42$ mag. We note that the first is in agreement with the distance modulus of the cluster. Kaluzny et al. (1997) assumed that B8 belongs to the cluster, finding $M_{V \max }=5.2 \mathrm{mag}$ and $M_{V \min }=7.6$, i.e., values very similar to ours. B8 is located at 4 ' from the center, and we can only conclude that the membership of this star is very probable.

\subsection{Contact binaries}

The simplest cases of eclipsing systems are the contact binaries (also named W UMa systems); they show short periods and continuous variability and therefore can be easily recognized and classified. We detected 29 of these variable stars; they have $P<0.40$ days and very well defined light curves. The complete list and the light curves are reported in the Appendix. Table 4 lists the stars likely belonging to NGC 6791 (see above); their light curves are shown in Fig. 8. The very short periods and the secondary minima occurring at $\phi \approx 0.5$ indicate binaries with circular orbits, as is also the case for stars with small amplitudes (in 7 cases we have amplitudes less than 0.20 mag: V3, V4, V5, V8, V23, V40 and 01441_8). The average error bar on the period estimates is of the order of $4-5 \times 10^{-5} \mathrm{~d}$.

However, we note that the stellar surfaces are not homogeneous since the maxima are often at different heights. Therefore, binarity and activity are probably combined here. In particular, the shape of the light curves of V4 (comparing RK96, M02 and our data) and V7 (comparing K93 and our data) have changed a lot; we suppose that stellar spots strongly modify the light curves. Proximity effects are also responsible for the large amplitudes observed for 01434_3 (Fig. 8, last panel) and 00766_5. We also found different periods for V118 (0.306321 d) and V124 (0.320143 d) compared to H05.

As for membership, the probabilities provided by Cudworth are $78 \%, 98 \%$ and $98 \%$ for V3, V4 and V5, respectively. Indeed, they are very close to the cluster center $(4.5,2 ! 1$ and $2 ! 8$, respectively). 

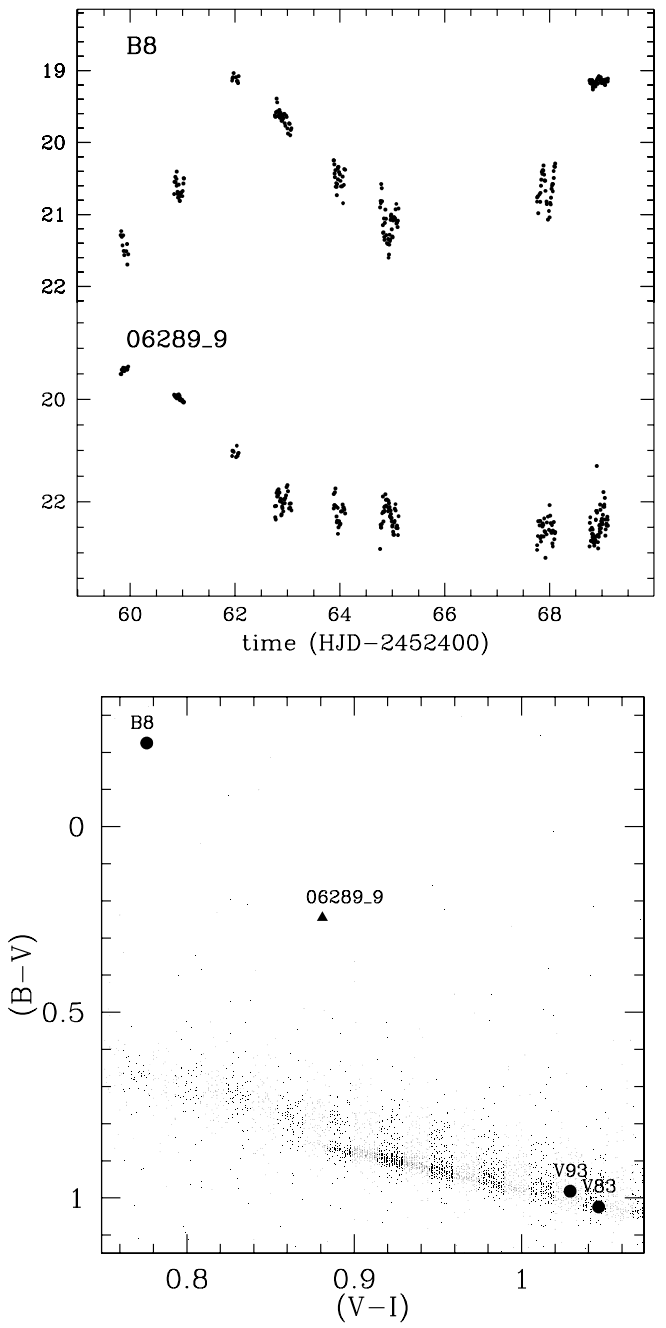

Fig. 7. Top: light curves of B8 (CV star) and 06289_9 (candidate CV). Bottom: positions in the two-colour diagram for two irregular stars (V83 and V93), for the cataclysmic variable B8 when in a low state, and for the new variable candidate 06289_9.

We suggest that $01441 \_8, \mathrm{~V} 118, \mathrm{~V} 8, \mathrm{~V} 117$ and V7 are also contact binaries belonging to NGC 6791. To further verify this hint, we calculated their distance by using the $P-L-C$ relation given by Rucinski (2003); they turn out to have distance moduli $(13.28,13.28,13.48,13.28$ and 13.18 , respectively) very similar to that of the cluster (13.35).

Moreover, these stars are located at similar angular distances from the cluster center $(6)^{\prime}, 7 ! 2,7 ! 1,7 ! 2$ and 6.3 , respectively). Figure 9 shows how the distance modulus of the cluster is in better agreement with those of the stars we proposed as cluster members than with those of the previously known members. Their positions in the CMDs (Fig. 10, filled circles) are similar to those of stars in the sample with $V<7.5$ whose parallaxes have been determinated by HIPPARCOS (Rucinski 2003). We also note that most of the cluster members are near the turnoff point.

\subsection{Eclipsing variables}

In the cases of detached or semi-detached eclipsing binaries the classification and membership tasks are different from the case of contact binaries. Table 5 lists the systems for which we could determine periods; their light curves are shown in Fig. 11. We

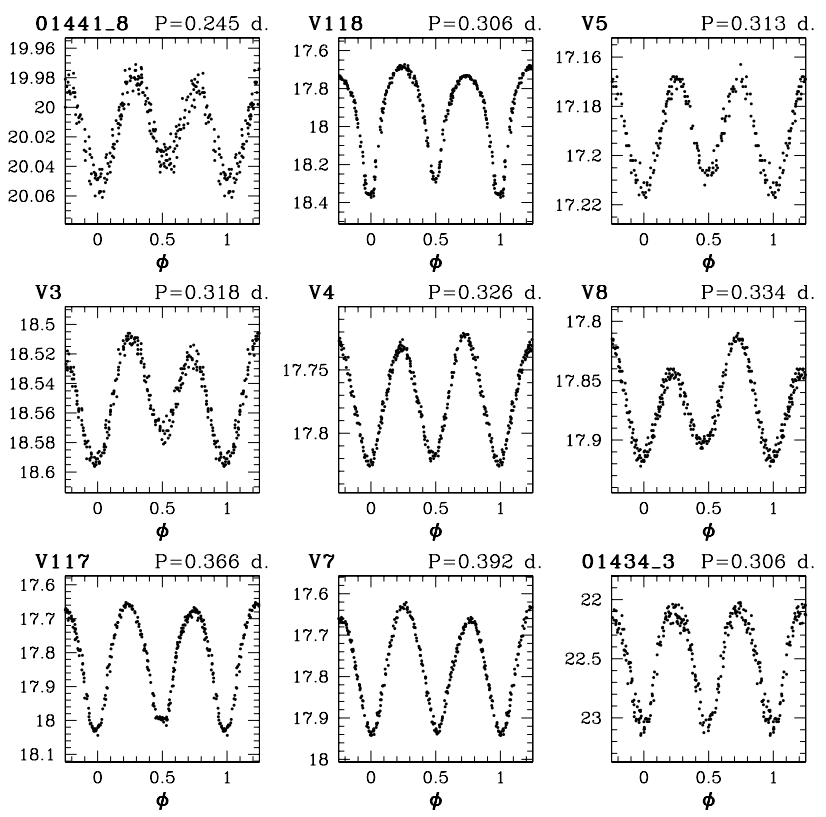

Fig. 8. The light curves of the contact binaries that are likely members of NGC 6791. The case of 01434_3 (amplitude much larger than $0.75 \mathrm{mag}$ ) is also shown in the last panel.

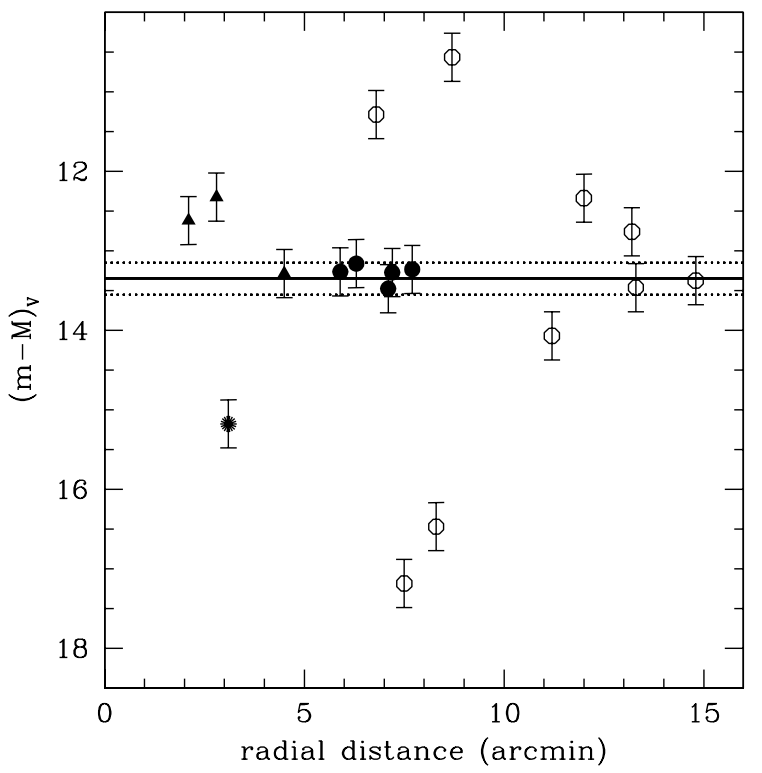

Fig. 9. The $m-M$ values calculated for contact binaries by means of the Rucinski (2003) $P-L-C$ relation plotted against the distance from the cluster center. Triangles indicate the stars whose membership has been proposed by M03, the filled circles the stars whose membership has been proposed by us, and the open circles the stars that we suggest do not belong to NGC 6791. The starred point indicates the star 09891_9 which does not belong to the cluster on the basis of the proper motion (B06). The $(m-M)_{V}$ value for the cluster (solid line) with an error bar of $\pm 0.20 \mathrm{mag}$ (dashed lines) is also shown.

still have short-period cases where we can reconstruct the complete light curve, as for the classical examples of $\beta$ Lyr variables (V29, 01558_5 and 00331_3). V9 is a more complicated $\beta$ Lyr system in which spots produce maxima with different heights. Indeed, it has been classified as an RS CVn variable by M05 and B03; they also observed a "shift of the modulation wave from 1995 to 2002 . 
Table 4. Coordinates and light curve parameters of the contact binaries (W UMa systems, EW) belonging to NGC 6791 . $V$ is the brightness at maximum and $T_{0}$ is the time of the primary minimum.

\begin{tabular}{lllllllllll}
\hline \hline Star & $\alpha_{2000}$ & $\delta_{2000}$ & $\begin{array}{c}V \\
{[\mathrm{mag}]}\end{array}$ & $\begin{array}{c}\langle B-V\rangle \\
{[\mathrm{mag}]}\end{array}$ & $\begin{array}{c}\langle V-I\rangle \\
{[\mathrm{mag}]}\end{array}$ & Ref. & $\begin{array}{l}T_{0} \\
{[\mathrm{HJD}-2452400]}\end{array}$ & $\begin{array}{l}\text { Period } \\
{[\mathrm{d}]}\end{array}$ & $\begin{array}{l}\text { Ampl. } \\
{[\mathrm{mag}]}\end{array}$ & Notes \\
\hline 01441_8 & 19.339422 & 37.778118 & 19.98 & 1.38 & & $\mathrm{k}$ & 63.073 & 0.24544 & 0.07 & likely memb. \\
V118 & 19.347500 & 37.651222 & 17.68 & 0.75 & 1.01 & $\mathrm{~s}$ & 59.912 & 0.30623 & 0.70 & likely memb. \\
V5 & 19.346258 & 37.813354 & 17.19 & 0.90 & 0.95 & $\mathrm{k}$ & 60.221 & 0.31274 & 0.05 & member $(98 \%)$ \\
V3 & 19.354380 & 37.769349 & 18.51 & 1.05 & 1.06 & $\mathrm{k}$ & 59.798 & 0.31764 & 0.09 & member $(78 \%)$ \\
V4 & 19.348396 & 37.806652 & 17.72 & 1.01 & & $\mathrm{k}$ & 59.591 & 0.32568 & 0.10 & member $(98 \%)$ \\
V8 & 19.341938 & 37.865810 & 17.81 & 0.79 & 0.88 & $\mathrm{k}$ & 59.896 & 0.33406 & 0.10 & likely memb. \\
V117 & 19.343433 & 37.665848 & 17.66 & 0.87 & 0.90 & $\mathrm{k}$ & 59.987 & 0.36644 & 0.38 & likely memb. \\
V7 & 19.340271 & 37.821892 & 17.63 & 0.93 & 0.86 & $\mathrm{k}$ & 59.820 & 0.39174 & 0.31 & likely memb. \\
\hline
\end{tabular}
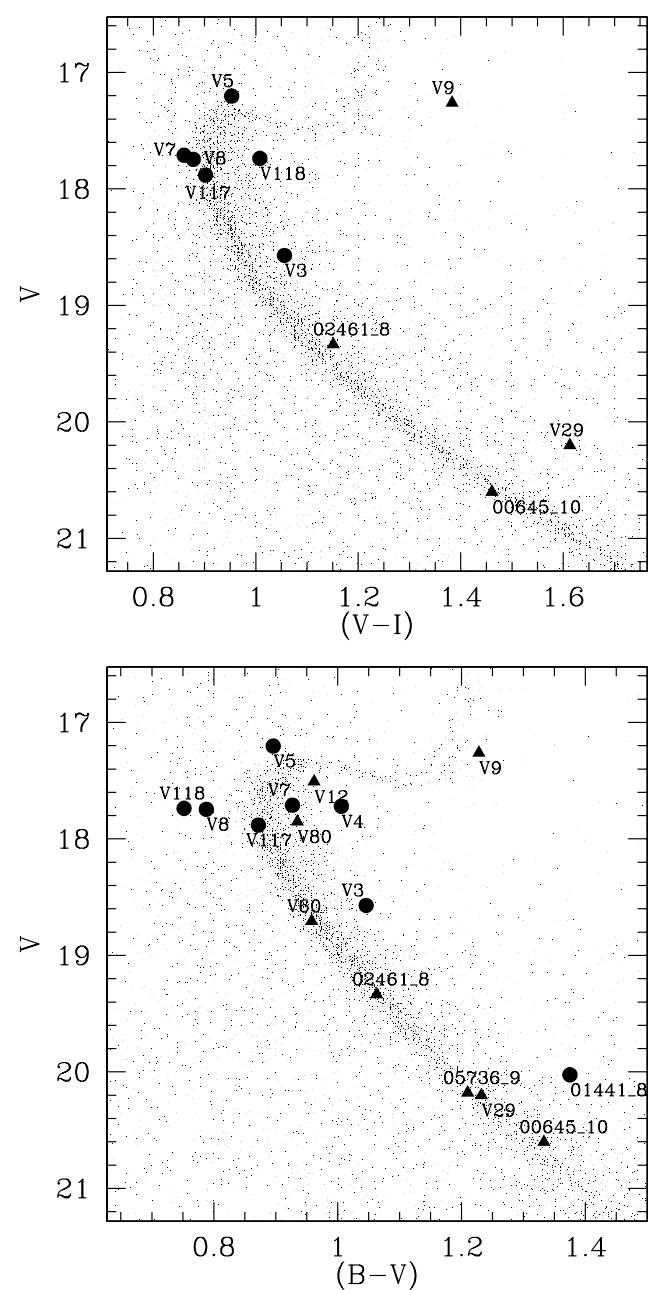

Fig. 10. The CMDs for the binary systems belonging to NGC 6791 . Filled circles are contact binaries (W UMa stars), triangles are detached or semi-detached systems ( $\beta$ Per or $\beta$ Lyr systems).

We note that our period for V119 is quite different from that given by H05 (0.1133 days); the new period makes this star an intermediate case between semi-detached and contact systems. Error bars on the periods in Table 5 are $\sim 10^{-4} \mathrm{~d}$ for $P<1.0 \mathrm{~d}$, $\sim 10^{-3} \mathrm{~d}$ for $1.0<P<2.0 \mathrm{~d}$ and a bit larger for $P>3.0 \mathrm{~d}$.

Some variables show very sharp eclipses and out-of-eclipse variability due to different levels of stellar activity (05736_9, 00645_10, V109,01393_1, V11 and V107; for the period of the latter star we prefer the longer of the two values given by M05).

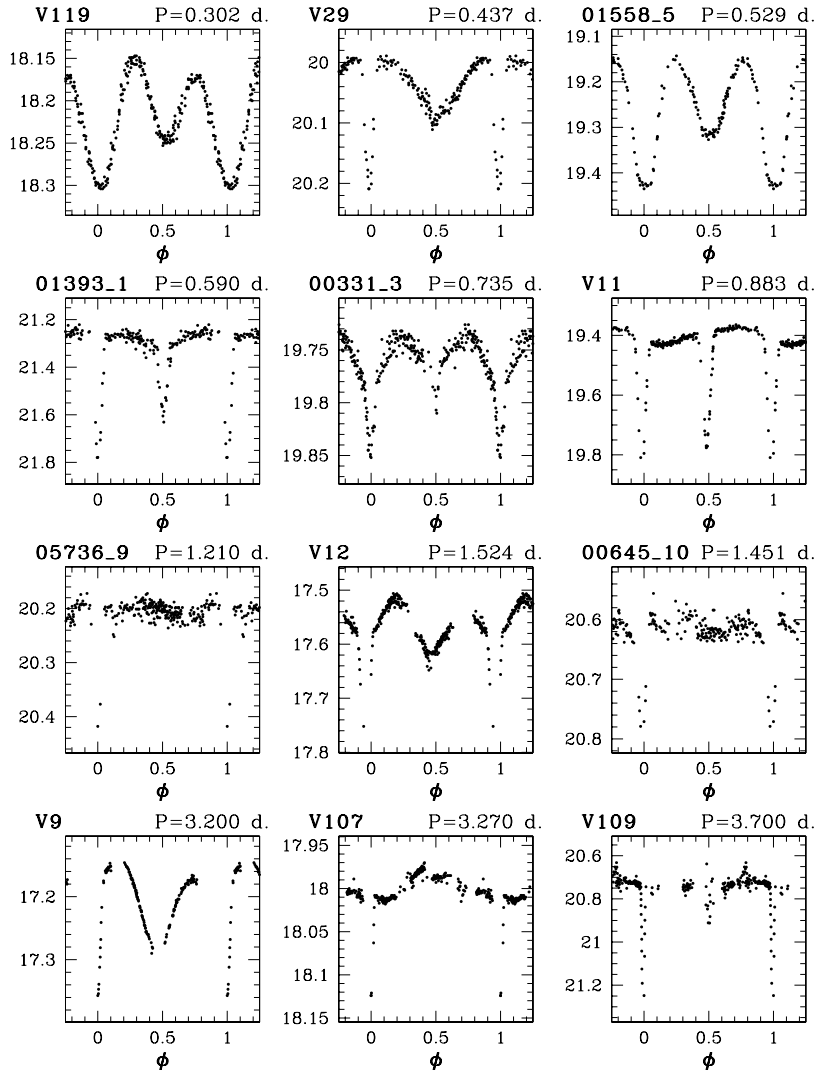

Fig. 11. The light curves of short-period detached or semi-detached eclipsing binaries.

In many cases we observed one eclipse only and we cannot give any value for the period, unless it has been given in the previous studies, as for the cluster member V80 (86\% on the basis of the Cudworth membership probability). We also note that the amplitude we observed in V80 is much larger than that reported by $\mathrm{B} 03$.

To establish the membership of these eclipsing systems is not an easy task, since binary effects should be taken into account when considering colors and magnitudes. However, on the basis of the distance from the cluster center and their position in the CMDs, we can argue that V60, 02461_8 (both singleevent eclipsing binaries), 05736_9, V29 and 00645_10 are very probable members. This hint is corroborated by the membership probabilities for V60 and 02461_8, which are $91 \%$ and $88 \%$ respectively. 
Table 5. Eclipsing variables with well defined light curves. EA stands for a $\beta$ Per system, EB for a $\beta$ Lyr one. $V$ is the brightness at maximum and $T_{0}$ is the time of the primary minimum. Also B4, V60 and 02461_8, whose parameters are reported in the Appendix, are possible cluster members. The last column shows the Cudworth membership probability (when available).

\begin{tabular}{llllccccclll}
\hline \hline Star & Type & $\alpha_{2000}$ & $\delta_{2000}$ & $\begin{array}{c}V \\
{[\mathrm{mag}]}\end{array}$ & $\begin{array}{c}\langle B-V\rangle \\
{[\mathrm{mag}]}\end{array}$ & $\begin{array}{c}\langle V-I\rangle \\
{[\mathrm{mag}]}\end{array}$ & $\begin{array}{l}\text { Ref. } \\
T_{0} \\
{[\mathrm{HJD}-2452400]}\end{array}$ & $\begin{array}{l}\text { Period } \\
{[\mathrm{d}]}\end{array}$ & $\begin{array}{l}\text { Ampl. } \\
{[\mathrm{mag}]}\end{array}$ & Notes \\
\hline V119 & EB & 19.351961 & 37.916328 & 18.15 & 1.13 & 1.33 & $\mathrm{k}$ & 59.879 & 0.30197 & 0.15 & member? \\
V29 & EB & 19.354796 & 37.751386 & 20.00 & 1.23 & 1.61 & $\mathrm{k}$ & 69.012 & 0.43662 & 0.22 & $\begin{array}{l}\text { likely memb. } \\
\text { 01558_5 }\end{array}$ \\
EB & 19.372697 & 37.953469 & 19.15 & & & & 69.028 & 0.52910 & 0.28 & likely non-memb. \\
01393_1 & EA & 19.329588 & 37.970392 & 21.23 & & & & 59.326 & 0.58998 & 0.56 & likely non-memb. \\
00331_3 & EB & 19.352367 & 37.864391 & 19.73 & 1.20 & 1.36 & $\mathrm{k}$ & 68.815 & 0.7347 & 0.13 & member? \\
V11 & EA & 19.342575 & 37.804802 & 19.38 & 0.96 & 1.22 & $\mathrm{k}$ & 67.875 & 0.8833 & 0.48 & member? \\
05736_9 & EA & 19.348484 & 37.721855 & 20.20 & 1.21 & & $\mathrm{k}$ & 68.333 & 1.210 & 0.29 & likely memb. \\
V12 & EB & 19.345259 & 37.849083 & 17.52 & 0.96 & & $\mathrm{k}$ & 64.103 & 1.524 & 0.06 & member $(96 \%)$ \\
00645_10 & EA & 19.354692 & 37.710104 & 20.60 & 1.33 & 1.46 & $\mathrm{k}$ & 60.893 & 1.451 & 0.20 & likely memb. \\
V9 & EB & 19.346634 & 37.777035 & 17.15 & 1.23 & 1.38 & $\mathrm{k}$ & $63.873:$ & 3.2 & $>0.2$ & member $(82 \%)$ \\
V107 & EA & 19.355068 & 37.761553 & 17.97 & 0.93 & 1.00 & $\mathrm{k}$ & 64.433 & 3.27 & 0.24 & member? \\
V109 & EA & 19.342716 & 37.793961 & 20.73 & 1.46 & 1.60 & $\mathrm{k}$ & 69.021 & 3.70 & 0.86 & member? \\
\hline
\end{tabular}

The special cases of V9 and B4 deserve attention. V9 is the binary closest to the center and its membership probability is $82 \%$. However, it looks a very evolved object in the CMD; its period ( $3.2 \mathrm{~d}$ ) and activity (see above) are also more typical for a Main Sequence star. Therefore, its membership is very doubtful.

The Cudworth membership probability for B4 is only $40 \%$, but in the CMDs B4 belongs to a little "clump" of very blue stars. This location is in agreement with the results of Liebert et al. (1994) and therefore B4 is likely a blue extanded horizontalbranch star belonging to NGC 6791. The star is classified by M02 and M03 (who consider it a non-member) as an eclipsing binary, but we note that the light curve could also result from a rotational modulation.

Other possible members are: V107, 00331_3, V109 and V11, considering that they are within 6.4 radius from the cluster center. The location in the CMDs of the eclipsing binaries belonging to NGC 6791 is shown in Fig. 10 (triangles).

\subsection{Rotational variables}

We found 89 variables whose light curves are characterized by small amplitude (usually less than $0.10 \mathrm{mag}$ ) and continuous variability. It is difficult to ascribe such variability to contact binaries undergoing grazing eclipses, since they should be less numerous than those having partial eclipses, since grazing eclipses occur only for a particular orientation of the orbital plane. Our hypothesis is that in most cases this variability results from spots carried by the stellar rotation; under this hypothesis, a large variety of light curves can be produced. Of course, we cannot rule out that a small fraction of these light curves might be actually generated by grazing eclipses.

The complete list of the rotational variables and their light curves is given in the Appendix. Here we discuss some examples. If the inclination of the rotational axis causes the progressive disappearance of the largest spots, the light curve displays continuous variation, which could be sine shaped in the simplest cases (a fraction of the spots is always visible; it can also produce Cepheid-like variability, as in the 001606_1 case, Fig. 12), or with a standstill (the hot or cold spots totally disappear; 00513_2 in Fig. 12) or, more commonly, it can be distorted by other spots besides the largest ones (00471_12 in Fig. 12). In cases of very active stars, a secondary wave also occurs (01175_5 in Fig. 12). Since the second wave often covers less than half of the period, these rotational variables can be distinguished from eclipsing

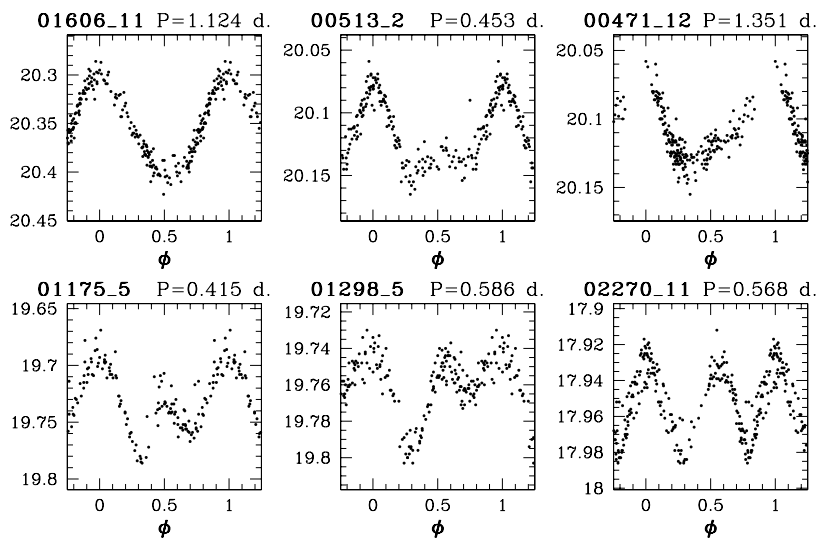

Fig. 12. The light curves of a small sample of rotational variables, illustrating the growing importance of the second wave.

binaries; we also note that the amplitude ratio between the first and second waves can be very different.

Also in the three cases in which the full amplitude is larger than 0.10 mag (V2, 02006_1 and 07483_9) rotational effects explain the observed features better than eclipses. For example, the light curves of V2 $(P=0.273 \mathrm{~d})$ and 01298_5 $(P=0.586 \mathrm{~d}$; see Fig. 12) show typical eclipsing binary behaviour, but the amplitudes, the periods, and, mainly, the asymmetries are more typical of a rotational effect. The case of 02270_11 is different (Fig. 12). Its light curve is very similar to that of a contact binary, but it does not repeat exactly, and unusual scatter is observed through the cycle. We also note that this non-repetitive behaviour of the light curves, due to the spot activity, is the reason why several variables stars show residual standard deviations higher than expected.

Our periods for V34, V37 and V38 are approximately half of those given by M02, since these authors classified these variables as ellipsoidal ones; the large amplitudes $(0.18,0.06$ and $0.13 \mathrm{mag}$ ) are more in favour of a variability resulting from large spots, rather than the purely geometrical effect of tidally distorted stars. We also note that V37 did not show any flare activity similar to that reported by M02 during our survey. We have also revised the classification of V16, considered an eclipsing binary by M02 and M03. 
Table 6. Rotational variables belonging to the cluster. $V$ is the mean brightness value. $T_{0}$ indicates the time of the maximum brightness. The last column shows the membership probability (when available) or our photometric membership. The two uncertain cases (V41 and V14) are also listed (see text).

\begin{tabular}{llllllllllll}
\hline \hline Star & Type & $\alpha_{2000}$ & $\delta_{2000}$ & $\begin{array}{c}V \\
{[\mathrm{mag}]}\end{array}$ & $\begin{array}{c}\langle B-V\rangle \\
{[\mathrm{mag}]}\end{array}$ & $\begin{array}{c}\langle V-I\rangle \\
{[\mathrm{mag}]}\end{array}$ & Ref. & $\begin{array}{l}T_{0} \\
{[\mathrm{HJD}-2452400]}\end{array}$ & $\begin{array}{l}\text { Period } \\
{[\mathrm{d}]}\end{array}$ & $\begin{array}{l}\text { Ampl. } \\
{[\mathrm{mag}]}\end{array}$ & Notes \\
\hline 04803_9 & RO1 & 19.347698 & 37.796043 & 21.81 & 1.33 & 1.84 & $\mathrm{~s}$ & 61.799 & 1.1034 & 0.17 & member (B06) \\
V82 & RO1 & 19.344366 & 37.793381 & 19.01 & 1.00 & 1.02 & $\mathrm{k}$ & 56.481 & 1.1568 & 0.04 & likely memb. \\
06553_9 & RO1 & 19.349134 & 37.672577 & 19.26 & 1.04 & 1.13 & $\mathrm{~s}$ & 61.421 & 1.3485 & 0.08 & likely memb. \\
01724_9 & RO1 & 19.344957 & 37.785362 & 20.73 & 1.29 & 1.69 & $\mathrm{~s}$ & 64.410 & 1.6130 & 0.17 & likely memb. \\
V38 & RO1 & 19.351021 & 37.768288 & 18.82 & 0.96 & & $\mathrm{k}$ & 55.630 & 1.96 & 0.13 & member $(92 \%)$ \\
03079_9 & RO1 & 19.346190 & 37.754753 & 19.23 & 1.14 & 1.26 & $\mathrm{k}$ & 66.630 & 2.640 & 0.07 & member $(93 \%)$ \\
V14 & RO1 & 19.347687 & 37.756874 & 18.58 & 0.93 & 1.05 & $\mathrm{k}$ & 55.933 & 5.45 & 0.05 & non-member $(0 \%)$ \\
V48 & RO1 & 19.352076 & 37.718506 & 17.51 & 0.88 & & $\mathrm{k}$ & 65.223 & 5.65 & 0.09 & member $(96 \%)$ \\
V17 & RO1 & 19.344135 & 37.817928 & 17.92 & 1.20 & 1.28 & $\mathrm{k}$ & 63.211 & 6.523 & 0.04 & member $(88 \%)$ \\
V51 & RO1 & 19.353382 & 37.748795 & 19.94 & 1.22 & 1.21 & $\mathrm{k}$ & 63.624 & 6.72 & 0.09 & likely memb. \\
V52 & RO1 & 19.355795 & 37.771935 & 17.49 & 0.88 & 0.88 & $\mathrm{k}$ & 64.345 & 7.06 & 0.03 & likely memb. \\
V53 & RO1 & 19.350233 & 37.743187 & 18.72 & 0.89 & 0.93 & $\mathrm{k}$ & 69.294 & 7.47 & 0.04 & member $(86 \%)$ \\
00436_3 & RO2 & 19.352205 & 37.878635 & 18.92 & 0.92 & 1.08 & $\mathrm{k}$ & 60.018 & 0.26601 & 0.04 & likely memb. \\
07483_9 & RO2 & 19.349997 & 37.746311 & 21.28 & 1.32 & 1.70 & $\mathrm{~s}$ & 60.465 & 0.4375 & 0.17 & likely memb. \\
V41 & RO2 & 19.347492 & 37.806892 & 19.09 & & & & 60.000 & 0.4798 & 0.07 & member $(77 \%)$ \\
V42 & RO2 & 19.350058 & 37.714867 & 19.51 & 1.05 & 1.16 & $\mathrm{k}$ & 60.323 & 0.5068 & 0.10 & member $(92 \%)$ \\
V16 & RO2 & 19.352108 & 37.802662 & 17.79 & 0.93 & 1.01 & $\mathrm{k}$ & 67.713 & 2.182 & 0.03 & member $(96 \%)$ \\
\hline
\end{tabular}

We count 33 rotational variables in the $10^{\prime}$-circle (i.e., $\left.0.105 \mathrm{star} / \operatorname{arcmin}^{2}\right)$ centered on the cluster, while we have 56 variables in the remaining $924-\operatorname{arcmin}^{2}$ area (i.e., $\left.0.061 \mathrm{star} / \operatorname{arcmin}^{2}\right)$. We have color indices $(B-V$ and/or $V-I)$ for 48 stars; 33 of them have a radial distance less than $10^{\prime}$ from the cluster center. We can confirm the membership for 6 stars having proper motion membership: V16, V38, V42, V48, V53 and 03079_9. We have no photometric indices for V41; however, it is at only $2^{\prime}$ from the cluster center and its Cudworth probability membership is $77 \%$. Therefore, we consider V41 a member. For V14 we have the opposite situation because this star is at $1^{\prime}$ from the cluster center and its positions in the CMDs agree very well with a membership, but the proper motion measurements rule out that it can be a cluster member (0\%) (see Fig. 13, V14 is displayed as a starred dot). As mentioned by M03, the positions of V17 in the CMDs are unusual. Other variables located below the subgiant branch like V17 were found in the open cluster M67 (Mathieu et al. 2003) and in the globular cluster 47 Tuc (Albrow et al. 2003). Probably these objects (named "red stragglers" or "sub-subgiant branch stars") are the result of some kind of mass exchange between the members of a binary system.

Putting the rotational variables without proper motion measurements on the CMDs we could infer that 8 stars are located on or close to the MS (represented with filled circles in Fig. 13); thus we suggest that these 8 stars belong to the cluster as well. Among the variables at greater distances, for three stars (01149_2,01122_4 and 00513_2, all located between 11' and $13^{\prime}$ ) the membership is doubtful, since their position in the CMDs is unclear. The other stars show apparent magnitudes and/or color indices too discrepant to be considered active MS stars belonging to NGC 6791.

When considering the variables without color indices, only two (V41 and 01874_2) are at less than 10' from the cluster center. We know that V41 is a probable cluster member (membership probability $77 \%$ ), but, at the moment, we have no valid reason to consider the other star as a member.

Table 6 lists the rotational variables we suggest as cluster members. The error bars on the period are $\sim 10^{-4} \mathrm{~d}$ for $P<1.0 \mathrm{~d}$, $\sim 10^{-3} \mathrm{~d}$ for $1.0<P<2.0 \mathrm{~d}, \sim 10^{-2} \mathrm{~d}$ for $2.0<P<5.0 \mathrm{~d}$; periods longer than $5.0 \mathrm{~d}$ are tentative. Figure 13 shows the
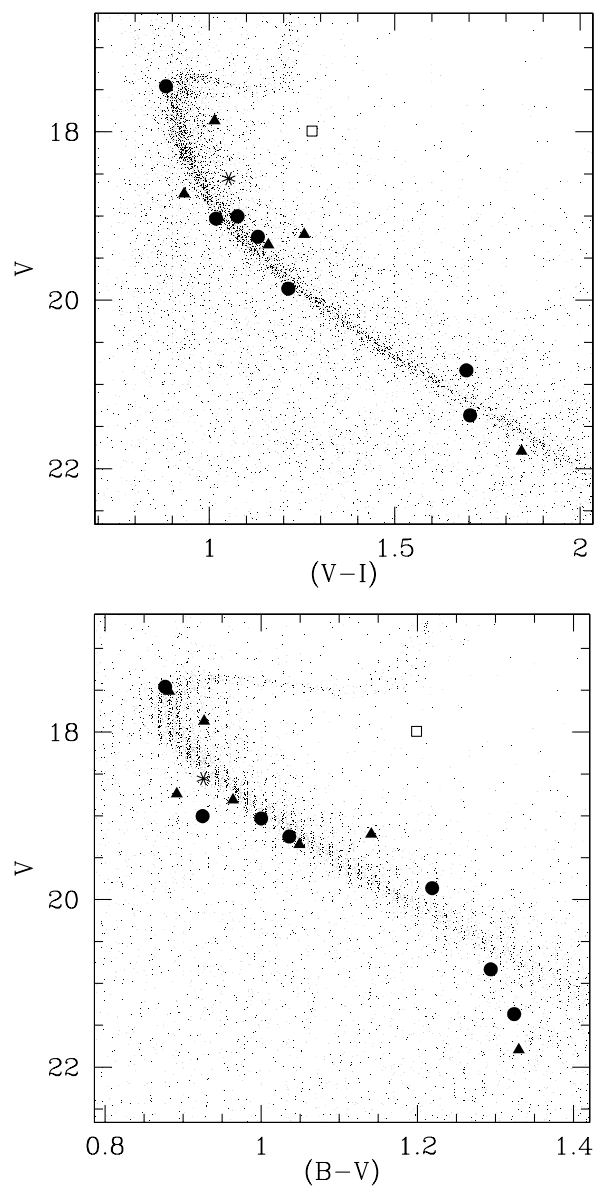

Fig. 13. $(V-I)-V$ and $(B-V)-V$ diagrams for NGC 6791. The rotational variables that we suggest may belong to the cluster are indicated with filled circles. Triangles: stars belonging to the cluster according to the Cudworth's membership; starred point: V14, open square: V17 (see text for details about these stars).

CMDs with the rotational variables belonging to the cluster (Table 6) clearly indicated. We rejected as cluster members 16 

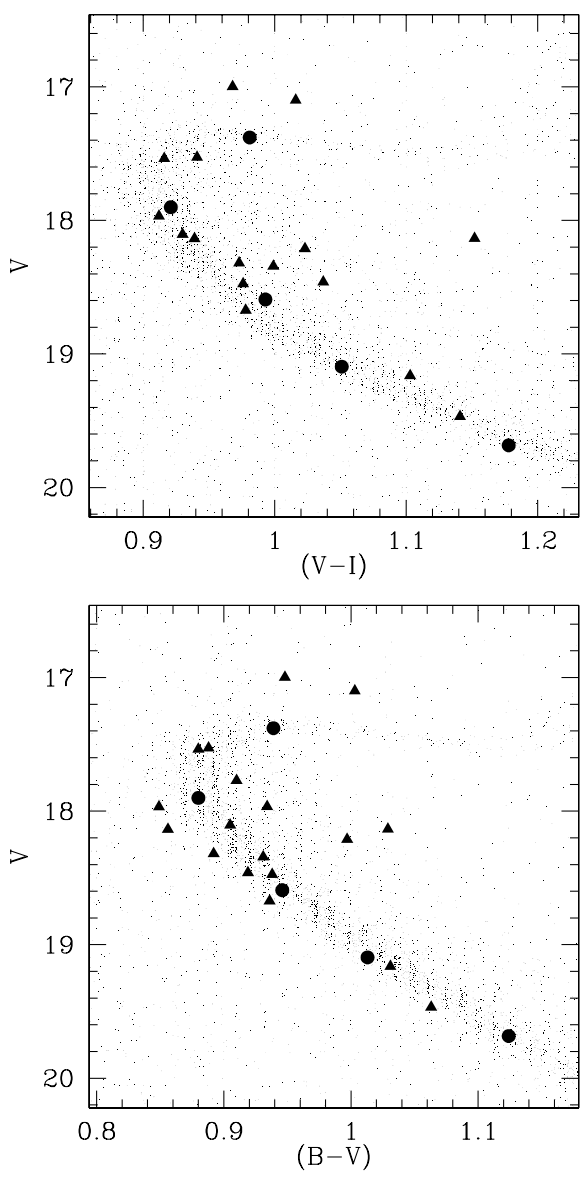

Fig. 14. $(B-V)-V$ and $(V-I)-V$ diagrams for NGC 6791. Filled circles: long-period variables that we suggest belong to the cluster. Filled triangles: long-period variables that belong to the cluster (membership probability higher than $76 \%$.)

stars out of 32 located within $10^{\prime}$ from the cluster center; i.e., we considered them to be stars of the Galactic field. We note that the resulting density of the Galactic field $\left(0.051 \mathrm{star} / \mathrm{arcmin}^{2}\right) \mathrm{su}-$ perimposed on the cluster is in good agreement with that of the surrounding galactic disk field (0.061 star/ $\operatorname{arcmin}^{2}$, see above), especially considering that Poisson statistics supply uncertainties around \pm 0.01 on the density values.

The stellar rotation and the activity level are both expected to be small for single stars as old as NGC 6791. Therefore we suggest that the rotational variables belonging to the cluster are likely short-period binaries, whose rotational velocity and activity level have been enhanced by the tidal synchronization.

\subsection{Long-period variables}

We detected numerous stars having different mean magnitudes on the different nights. Their behaviours are more diversified than those of the stars we considered as spurious on the basis of their close similarities. The resulting power spectra are dominated by terms at very low frequencies, corresponding to periods often much longer than $10 \mathrm{~d}$. These periods cannot be evaluated in a precise way, being comparable or, more frequently, longer than our time baseline. Therefore, we can only argue that these stars are variables, either in a periodic or in an irregular way. Since we detected many spotted stars, it is quite obvious to think that most of these long-period variables are spotted stars having a rotational periods longer than $10 \mathrm{~d}$. The mean amplitude of

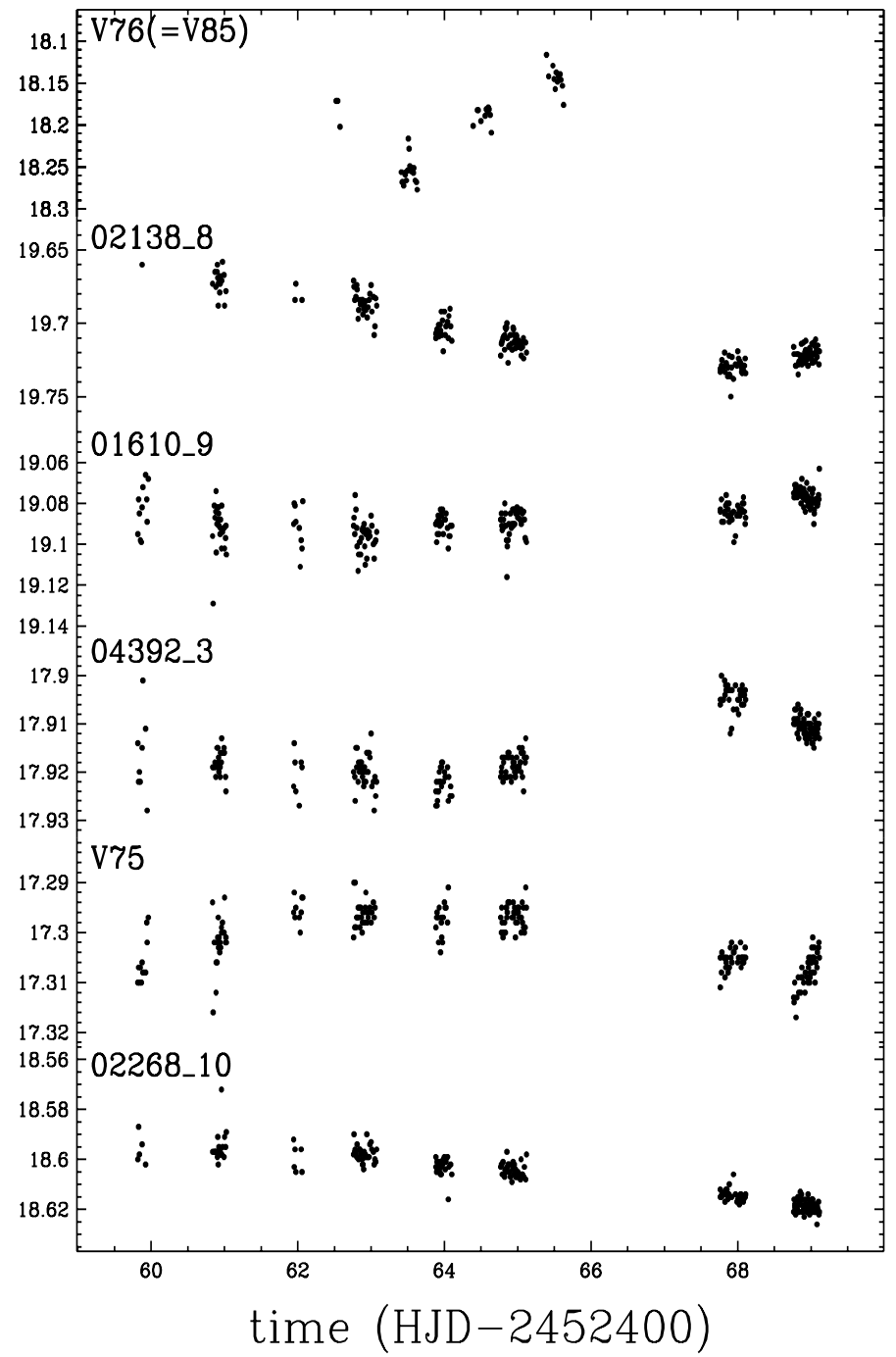

Fig. 15. Light curve of the suspect "red straggler" V76 $\equiv$ V85 and the five stars that we suggest may belong to the cluster.

these stars is about $0.02 \mathrm{mag}$, except for 5 stars whose amplitude exceeds 0.1 mag.

Among the long-period variables, we used the Cudworth probabilities to establish the membership of 18 stars. In order to roughly estimate the membership of the remaining long-period variables we checked their locations in the CMDs, in the cases where at least one color is available. We suggest that 5 stars are likely members of NGC 6791: 02138_8, 01610_9, 04392_3, V75 and 02268_10 (see Fig. 14). They lie along the MS or the red-giant branch and, furthermore, they are all located at distances smaller than 8.5, from the cluster center. Looking at the position of the variable V76 $\equiv$ V85 (memberships: 97\%) in both CMDs, we suggest that this star could be similar to the "subsubgiant branch" star V17. In Fig. 15 we show its light curve and those of the 5 stars that we suspect to belong to the cluster. Table 7 lists the long-period variables we suggest as cluster members; the entire sample is listed in the Appendix.

\section{Conclusions}

Our wide-field survey of NGC 6791 for the planetary-transit search allowed us to discover 260 new variable stars. When considering the membership probabilities given by Cudworth and B06, 13 of them belong to the cluster and one star (09831_9) is 
Table 7. Long period variable stars that are likely members of NGC 6791 (ordered by increasing right ascension). Column 9 shows the membership probability (when available) or our photometric membership.

\begin{tabular}{|c|c|c|c|c|c|c|c|c|c|}
\hline Star & $\alpha_{2000}$ & $\delta_{2000}$ & $\begin{array}{c}V \\
{[\mathrm{mag}]}\end{array}$ & $\begin{array}{c}\langle B-V\rangle \\
{[\mathrm{mag}]}\end{array}$ & $\begin{array}{c}\langle V-I\rangle \\
{[\mathrm{mag}]}\end{array}$ & Ref. & $\begin{array}{l}\text { Ampl. } \\
\text { [mag] }\end{array}$ & Notes & $\begin{array}{l}\text { Distance } \\
\text { arcmin }\end{array}$ \\
\hline $02138 \_8$ & 19.341864 & 37.749653 & 19.68 & 1.12 & 1.18 & $\mathrm{k}$ & 0.06 & likely memb. & 4.6 \\
\hline 02444_8 & 19.342766 & 37.810604 & 18.34 & 0.93 & 1.00 & $\mathrm{k}$ & 0.02 & member $(78 \%)$ & 4.4 \\
\hline 00510_9 & 19.343704 & 37.796719 & 18.67 & 0.94 & 0.98 & $\mathrm{k}$ & 0.03 & member $(83 \%)$ & 3.4 \\
\hline 01610_9 & 19.344818 & 37.740486 & 19.09 & 1.01 & 1.05 & $\mathrm{k}$ & 0.02 & likely memb. & 3.0 \\
\hline V94 & 19.345139 & 37.743549 & 17.54 & 0.88 & 0.92 & $\mathrm{k}$ & 0.03 & member $(90 \%)$ & 2.7 \\
\hline V95 & 19.345295 & 37.792412 & 19.16 & 1.03 & 1.10 & $\mathrm{k}$ & 0.07 & member $(93 \%)$ & 2.3 \\
\hline V56(=V96) & 19.345908 & 37.763525 & 17.01 & 0.95 & 0.97 & $\mathrm{k}$ & 0.04 & member $(98 \%)$ & 1.6 \\
\hline 04392_3 & 19.345982 & 37.870571 & 17.90 & 0.88 & 0.92 & $\mathrm{k}$ & 0.02 & likely memb. & 6.1 \\
\hline V75 & 19.346651 & 37.766308 & 17.38 & 0.94 & 0.98 & $\mathrm{~s}$ & 0.01 & likely memb. & 1.0 \\
\hline 04133_9 & 19.347109 & 37.777020 & 18.47 & 0.94 & 0.98 & $\mathrm{k}$ & 0.04 & member $(98 \%)$ & 0.7 \\
\hline V76(=V85) & 19.347192 & 37.764169 & 18.19 & 1.03 & 1.15 & $\mathrm{k}$ & 0.11 & member $(97 \%)$ & 0.8 \\
\hline V86 & 19.347258 & 37.808815 & 19.44 & 1.06 & 1.14 & $\mathrm{k}$ & 0.03 & member $(83 \%)$ & 2.3 \\
\hline V87 & 19.347996 & 37.749668 & 18.12 & 0.91 & 0.93 & $\mathrm{k}$ & 0.03 & member $(98 \%)$ & 1.3 \\
\hline 05740_9 & 19.348534 & 37.808022 & 17.96 & 0.93 & & $\mathrm{k}$ & 0.03 & member $(95 \%)$ & 2.2 \\
\hline 06725_9 & 19.349329 & 37.724495 & 17.77 & 0.91 & & $\mathrm{k}$ & 0.02 & member $(91 \%)$ & 3.0 \\
\hline 06796_9 & 19.349415 & 37.769268 & 18.46 & 0.92 & 1.04 & $\mathrm{k}$ & 0.03 & member $(92 \%)$ & 1.0 \\
\hline V90 & 19.349686 & 37.746449 & 18.11 & 0.86 & 0.94 & $\mathrm{k}$ & 0.01 & member $(94 \%)$ & 1.9 \\
\hline 07680_9 & 19.350193 & 37.718224 & 18.32 & 0.89 & 0.97 & $\mathrm{k}$ & 0.03 & member $(98 \%)$ & 3.5 \\
\hline V31 & 19.350683 & 37.785912 & 17.12 & 1.00 & 1.02 & $\mathrm{k}$ & 0.01 & member $(97 \%)$ & 2.1 \\
\hline 09376_9 & 19.351952 & 37.831886 & 18.21 & 1.00 & 1.02 & $\mathrm{k}$ & 0.01 & member $(92 \%)$ & 4.6 \\
\hline 09611_9 & 19.352139 & 37.773365 & 17.97 & 0.85 & 0.91 & $\mathrm{k}$ & 0.01 & member $(76 \%)$ & 2.9 \\
\hline V58 & 19.354042 & 37.801240 & 17.52 & 0.89 & 0.94 & $\mathrm{k}$ & 0.05 & member $(87 \%)$ & 4.6 \\
\hline 02268_10 & 19.359475 & 37.731544 & 18.59 & 0.95 & 0.99 & $\mathrm{k}$ & 0.02 & likely memb. & 8.5 \\
\hline
\end{tabular}

not member. On the basis of the distances from the cluster center and the positions in the CMDs, we suggest that another 11 stars are likely members, for 22 stars the membership is doubtful, and 137 stars are likely non-members. No photometric or kinematic data are available for 76 stars.

The variable star content of the cluster is very similar to that of the surrounding Galactic environment: in both samples we find rotational variables, contact and eclipsing systems. Contact binaries and rotational variables belonging to the cluster have the same characteristics as those located in the surrounding Galactic field. No evidence of pulsating variables has been found in NGC 6791, but this is not surprising, since it is a very evolved cluster and stars located in the instability strip or hotter pulsators have already left the MS.

The discovery of the new cataclysmic variable 06289_9 in addition to B8 and V15 adds another peculiarity to NGC 6791 , making it unusual among the open clusters.

Acknowledgements. We are grateful to Kyle Cudworth kindly providing us with preliminary cluster membership probabilities. We also acknowledge Prof. Antonio Bianchini for his suggestions about the characteristics of the candidate cataclysmic variable and Giovanni Carraro for his useful comments. We thank the referee, Dr. J. Kaluzny, for his detailed report and useful comments. This work was funded by COFIN 2004 "From stars to planets: accretion, disk evolution and planet formation" by MIUR and by PRIN 2006 "From disk to planetary systems: understanding the origin and demographics of solar and extrasolar planetary systems" by INAF.

\section{References}

Albrow, M. D., Gilliland, R. L., Brown, T. M., et al. 2001, ApJ, 559, 1060 Anthony-Twarog, B. J., Twarog, B. A., \& Mayer, L. 2006, preprint [arXiv:astro-ph/0612549]

Bedin, L. R., Salaris, M., Piotto, G., et al. 2005, ApJ, 624, 45
Bedin, L. R., Piotto, G., Carraro, G., King, I. R., \& Anderson, J. 2006, A\&A, 460, 27 (B06)

Bruntt, H., Grundahl, F., Tingley, B., et al. 2003, A\&A, 410, 323 (B03)

Carpino, M., Milani, A., \& Nobili, A. M. 1987, A\&A, 181, 182 Carraro, G., Villanova, S., Demarque, P., et al. 2006, ApJ, 643, 1151

Chaboyer, B., Green, E. M., \& Liebert, J. 1999, AJ, 117, 1360

Ferraz-Mello, S. 1981, AJ, 86, 4

Gratton, R., Bragaglia, A., Carretta, E., \& Tosi, M. 2006, ApJ, 642, 462

Hartman, J. D., Stanek, K. Z., Gaudi, B. S. et al. 2005, AJ, 130, 2241 (H05)

Kaluzny, J. 2003, Acta Astron., 53, 51 (K03)

Kaluzny, J., \& Rucinski, S. M. 1993, MNRAS, 265, 34 (KR93)

Kaluzny, J., \& Rucinski, S. M. 1995, A\&AS, 114, 1

Kaluzny, J., \& Udalski, A. 1992, Acta Astron., 42, 29

Kaluzny, J., Stanek, K. Z., Garnavich, P. M., \& Challis, P. 1997, ApJ, 491, 153

King, I. R., Bedin, L. R., Piotto, G., Cassisi, S., \& Anderson, J. 2005, AJ, 130, 626

Kòvacs, G., Zucker, S., \& Mazeh, T. 2002, A\&A, 391, 369

Liebert, J., Saffer, R. A., \& Green, E. M. 1994, AJ, 107, 1408

Lomb, N. R. 1976, Astrophysics and Space Science, 39, 447

Mathieu, R. D., van den Berg, M., Torres, G., et al. 2003, AJ, 125, 246

McNamara, D. H. 2000, in Delta Scuti and Related Starsn ed. M. Breger, \& M. H. Montgomery, ASP Conf. Ser., 210, 373

Mochejska, B. J., Stanek, K. Z., Sasselov, D. D., \& Szentgyorgyi, A. H. 2002, AJ, 123, 3460 (M02)

Mochejska, B. J., Stanek, K. Z., \& Kaluzny, J. 2003, AJ, 125, 3175 (M03)

Mochejska, B. J., Stanek, K. Z., Szentgyorgyi, A. H., et al. 2005, AJ, 129, 2856 (M05)

Montalto, M., Piotto, G., Desidera, S., et al. 2007, A\&A, 470, 1137

Poretti, E. 2001, A\&A, 371, 986

Rucinski, S. M. 1995, PASP, 107, 648

Rucinski, S. M. 2003, New Astron. Rev., 48, 703

Rucinski, S. M., Kaluzny, J., \& Hilditch, R. W. 1996, MNRAS, 282, 705 (RK96) Stellingwerf, R. F. 1978, ApJ, 224, 953

Stetson, P. B., Bruntt, H., \& Grundahl, F. 2003, PASP, 115, 413

Tammann, G. A, Sandage, A., \& Reindl, B. 2003, A\&A, 404, 423

Vanicek, P. 1971, Astrophysics and Space Science, 12, 10

Warner, B. 1995, Cataclysmic Variable Stars (Cambridge: Cambridge Univ. Press) 
F. De Marchi et al.: Variable stars in the open cluster NGC 6791 and its surrounding field, Online Material $p 1$

\section{Online Material}




\section{Appendix A: List of identified variables}

This Appendix includes the full list of the identified variables, separated according to our classification:

1. Pulsating, irregular and cataclysmic variables: Table A.1.

2. EW-type stars: Table A.2, Fig. A.1.

3. EA/EB-type stars: Table A.3.

4. Single-wave rotational variables: Table A.4, Figs. A.2, A.3.

5. Double-wave rotational variables: Table A.5, Fig. A.4.

6. Long-period variables: Table A.6.

Into the tables, for each star we give the name (a five-digit number followed by the chip number which the star belongs), coordinates, photometric data (always the $V$ mag, $B-V$ color when available), informations about the variability $\left(T_{0}\right.$, period, amplitude), distance from the center (in arcmin) and finally the numerical value of the Cudworth's membership probability (reported in the column "Memb.").

In most cases, when membership probabilities were not available, in the same column the label "m" means that we retain the star belonging to the cluster, while " $m$ ?" and " $\mathrm{nm}$ " mean "uncertain membership" and "likely non-member" respectively. The label "nd1" means that no photometric data are available to advance hypothesis about the membership, but the star is located nearer than $10^{\prime}$ from the center of the cluster. Finally "nd2" means that no photometric data are available and the star is located further than $10^{\prime}$ from the center; in this case we strongly suggest that the star does not belongs to the cluster. 
F. De Marchi et al.: Variable stars in the open cluster NGC 6791 and its surrounding field, Online Material p 3

Table A.1. Pulsating, irregular and cataclysmic variables. $V$ is the minimum brightness for CVs and irregular, the mean brightness for pulsating variables. $T_{0}$ is the time of maximum brightness for pulsating stars.

\begin{tabular}{|c|c|c|c|c|c|c|c|c|c|c|c|c|}
\hline Star & Type & $\alpha_{2000}$ & $\delta_{2000}$ & $\begin{array}{c}V \\
{[\mathrm{mag}]}\end{array}$ & $\begin{array}{c}\langle B-V\rangle \\
{[\mathrm{mag}]}\end{array}$ & $\begin{array}{c}\langle V-I\rangle \\
{[\mathrm{mag}]}\end{array}$ & Ref. & $\begin{array}{l}T_{0} \\
\text { [HJD-2 452 400] }\end{array}$ & $\begin{array}{l}\text { Period } \\
\text { [d] }\end{array}$ & $\begin{array}{l}\text { Ampl. } \\
\text { [mag] }\end{array}$ & Memb. & $\begin{array}{l}\text { Distance } \\
\text { [arcmin] }\end{array}$ \\
\hline V123 & HADS & 19.362064 & 37.666034 & 17.08 & 0.45 & & $\mathrm{k}$ & 59.559 & 0.06026 & 0.14 & $\mathrm{~nm}$ & 11.8 \\
\hline 01497_12 & HADS & 19.379083 & 37.812419 & 16.06 & & & & 59.528 & 0.07227 & 0.40 & nd2 & 22.2 \\
\hline 00311_7 & SXPhe & 19.324628 & 37.716768 & 23.17 & & & & 59.605 & 0.10443 & 0.10 & nd2 & 17.0 \\
\hline 00224_10 & SXPhe & 19.353639 & 37.710163 & 21.72 & 0.71 & 1.06 & $\mathrm{~s}$ & 59.801 & 0.12261 & 0.20 & $\mathrm{~nm}$ & 5.4 \\
\hline $03653 \_3$ & RRc & 19.347147 & 37.992413 & 17.21 & 0.57 & 0.58 & $\mathrm{k}$ & 59.937 & 0.32654 & 0.39 & $\mathrm{~nm}$ & 13.3 \\
\hline 00345_1 & RRab & 19.325082 & 37.964170 & 18.28 & & & & 60.151 & 0.57866 & 0.72 & nd2 & 20.0 \\
\hline V92 & IRR & 19.350754 & 37.766876 & 18.10 & 0.91 & & $\mathrm{k}$ & & & 0.10 & $\mathrm{~m}$ & 1.9 \\
\hline V83 & IRR & 19.346220 & 37.737232 & 19.10 & 1.02 & 1.05 & $\mathrm{k}$ & & & 0.07 & $\mathrm{~m}$ & 2.4 \\
\hline V93 & IRR & 19.351452 & 37.785687 & 18.12 & 0.98 & 1.03 & $\mathrm{~s}$ & & & 0.04 & $\mathrm{~m}$ & 2.6 \\
\hline $\mathrm{V} 15(=\mathrm{B} 7)$ & $\mathrm{CV}$ & 19.352057 & 37.799019 & 18.26 & 0.20 & & $\mathrm{k}$ & & & 0.06 & 98 & 3.3 \\
\hline B8 & $\mathrm{CV}$ & 19.343262 & 37.747833 & 20.64 & -0.23 & 0.78 & $\mathrm{k}$ & & & 2.27 & $\mathrm{~m}$ & 3.7 \\
\hline 06289_9 & $\mathrm{CV}$ & 19.348976 & 37.770355 & 22.80 & 0.25 & 0.88 & $\mathrm{~s}$ & & & 3.10 & m (B06) & 0.7 \\
\hline
\end{tabular}

Table A.2. Contact binaries; $V$ is the brightness at maximum and $T_{0}$ is the time of the primary minimum.

\begin{tabular}{|c|c|c|c|c|c|c|c|c|c|c|c|}
\hline Star & $\alpha_{2000}$ & $\delta_{2000}$ & $\begin{array}{c}V \\
{[\mathrm{mag}]}\end{array}$ & $\begin{array}{c}\langle B-V\rangle \\
{[\mathrm{mag}]}\end{array}$ & $\begin{array}{c}\langle V-I\rangle \\
{[\mathrm{mag}]}\end{array}$ & Ref. & $\begin{array}{l}T_{0} \\
\text { [HJD-2 452 400] }\end{array}$ & $\begin{array}{l}\text { Period } \\
\text { [d] }\end{array}$ & $\begin{array}{l}\text { Ampl. } \\
\text { [mag] }\end{array}$ & Memb. & $\begin{array}{l}\text { Distance } \\
\text { [arcmin] }\end{array}$ \\
\hline V122 & $\begin{array}{l}9.360729 \\
\end{array}$ & 37.641436 & 20.92 & & & & 59.614 & 0.22883 & 0.58 & nd2 & 11.9 \\
\hline V115 & 19.330646 & 37.975902 & 20.70 & & & & 59.473 & 0.23636 & 0.24 & $\mathrm{nd} 2$ & 17.4 \\
\hline 01407_8 & 19.339256 & 37.701576 & 21.52 & 0.64 & 1.25 & $\mathrm{k}$ & 59.447 & 0.24155 & 0.82 & $\mathrm{~nm}$ & 7.5 \\
\hline 01150_4 & 19.357227 & 37.962177 & 18.58 & 1.07 & 0.93 & $\mathrm{k}$ & 59.455 & 0.24510 & 0.27 & $\mathrm{~nm}$ & 13.2 \\
\hline 01441_8 & 19.339422 & 37.778118 & 19.98 & 1.38 & & $\mathrm{k}$ & 63.073 & 0.24544 & 0.07 & $\mathrm{~m}$ & 6.2 \\
\hline 00144_2 & 19.334044 & 38.030701 & 19.19 & & & & 59.457 & 0.24780 & 0.77 & $\mathrm{nd} 2$ & 18.5 \\
\hline 01670_10 & 19.357625 & 37.681442 & 16.64 & 1.14 & 1.24 & $\mathrm{k}$ & 59.729 & 0.25807 & 0.49 & $\mathrm{~nm}$ & 8.7 \\
\hline V121 & 19.358063 & 37.932346 & 17.24 & 0.81 & 0.84 & $\mathrm{k}$ & 59.619 & 0.26742 & 0.71 & $\mathrm{~nm}$ & 12.0 \\
\hline 02291_11 & 19.371624 & 37.795464 & 19.15 & & & & 59.582 & 0.26774 & 0.29 & $\mathrm{nd} 2$ & 16.8 \\
\hline V23 & 19.338614 & 37.787781 & 16.92 & 1.04 & 1.23 & $\mathrm{k}$ & 59.915 & 0.27180 & 0.07 & $\mathrm{~nm}$ & 6.8 \\
\hline V25 & 19.328426 & 37.713237 & 18.50 & & & & 59.772 & 0.27730 & 0.56 & nd2 & 14.4 \\
\hline 00665_12 & 19.375697 & 37.713244 & 18.89 & & & & 59.472 & 0.28369 & 0.41 & nd2 & 20.0 \\
\hline 09831_9 & 19.352356 & 37.780907 & 20.55 & 1.09 & 1.19 & $\mathrm{~s}$ & 59.662 & 0.29488 & 0.3 & nm (B06) & 3.1 \\
\hline 01434_3 & 19.350643 & 37.985512 & 22.10 & & & & 59.831 & 0.30581 & 0.97 & nd2 & 13.0 \\
\hline V118 & 19.347500 & 37.651222 & 17.68 & 0.75 & 1.01 & $\mathrm{~s}$ & 59.912 & 0.30623 & 0.70 & $\mathrm{~m}$ & 7.2 \\
\hline 00721_11 & 19.365737 & 37.713858 & 15.53 & & & & 59.491 & 0.31008 & 0.26 & $\mathrm{nd} 2$ & 13.1 \\
\hline 01701_2 & 19.341944 & 38.017998 & 18.72 & & & & 59.534 & 0.31201 & 0.51 & nd2 & 15.4 \\
\hline V5 & 19.346258 & 37.813354 & 17.19 & 0.90 & 0.95 & $\mathrm{k}$ & 60.221 & 0.31274 & 0.05 & 98 & 2.8 \\
\hline V3 & 19.354380 & 37.769349 & 18.51 & 1.05 & 1.06 & $\mathrm{k}$ & 59.798 & 0.31764 & 0.09 & 78 & 4.5 \\
\hline 02030_4 & 19.353487 & 38.009422 & 19.04 & 1.19 & 1.42 & $\mathrm{k}$ & 59.614 & 0.31797 & 0.33 & $\mathrm{~nm}$ & 14.8 \\
\hline V124 & 19.365150 & 37.681544 & 17.62 & 0.70 & 1.07 & $\mathrm{k}$ & 59.855 & 0.32014 & 0.58 & $\mathrm{~nm}$ & 13.3 \\
\hline 00766_5 & 19.367585 & 37.943904 & 22.31 & & & & 59.466 & 0.32363 & 0.78 & $\mathrm{nd} 2$ & 17.3 \\
\hline V4 & 19.348396 & 37.806652 & 17.72 & 1.01 & & $\mathrm{k}$ & 59.591 & 0.32568 & 0.10 & 98 & 2.1 \\
\hline V27 & 19.336275 & 37.648720 & 18.47 & 0.82 & 1.29 & $\mathrm{k}$ & 59.763 & 0.33170 & 0.84 & $\mathrm{~nm}$ & 11.2 \\
\hline V8 & 19.341938 & 37.865810 & 17.81 & 0.79 & 0.88 & $\mathrm{k}$ & 59.896 & 0.33406 & 0.10 & $\mathrm{~m}$ & 7.1 \\
\hline V101 & 19.351563 & 37.640388 & 19.94 & 0.56 & & $\mathrm{k}$ & 59.798 & 0.33480 & 0.29 & $\mathrm{~nm}$ & 8.3 \\
\hline V117 & 19.343433 & 37.665848 & 17.66 & 0.87 & 0.90 & $\mathrm{k}$ & 59.987 & 0.36644 & 0.38 & $\mathrm{~m}$ & 7.2 \\
\hline V7 & 19.340271 & 37.821892 & 17.63 & 0.93 & 0.86 & $\mathrm{k}$ & 59.820 & 0.39174 & 0.31 & $\mathrm{~m}$ & 6.3 \\
\hline V40 & 19.327495 & 37.616839 & 19.67 & & & & 60.101 & 0.39750 & 0.16 & nd2 & 17.3 \\
\hline
\end{tabular}


F. De Marchi et al.: Variable stars in the open cluster NGC 6791 and its surrounding field, Online Material p 4 $\begin{array}{llll}V 122 & P=0.229 d\end{array}$ V115 $\quad P=0.236 d \quad 014078 \quad P=0.242 d$ $01150 \_4 \quad \mathrm{P}=0.245 \mathrm{~d} . \quad 01441 \_8 \quad \mathrm{P}=0.245 \mathrm{~d}$.

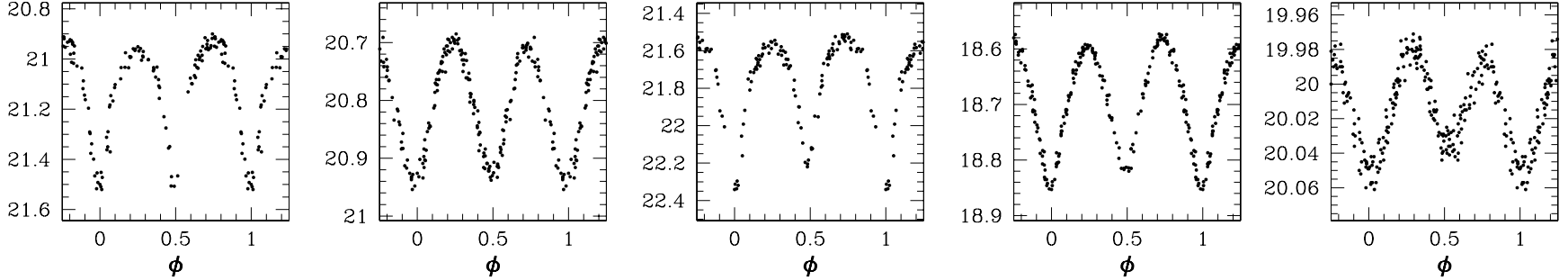

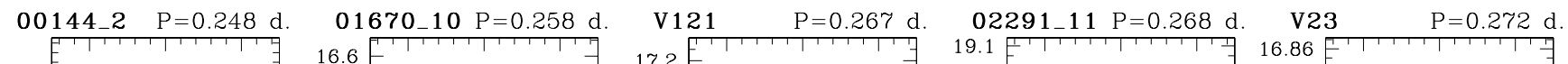

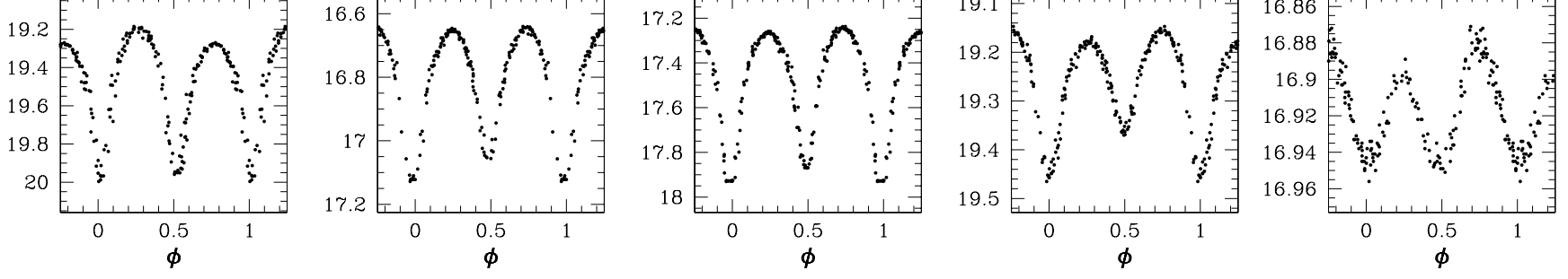

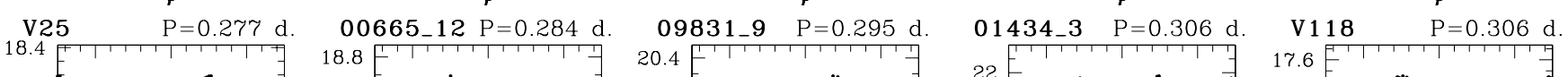
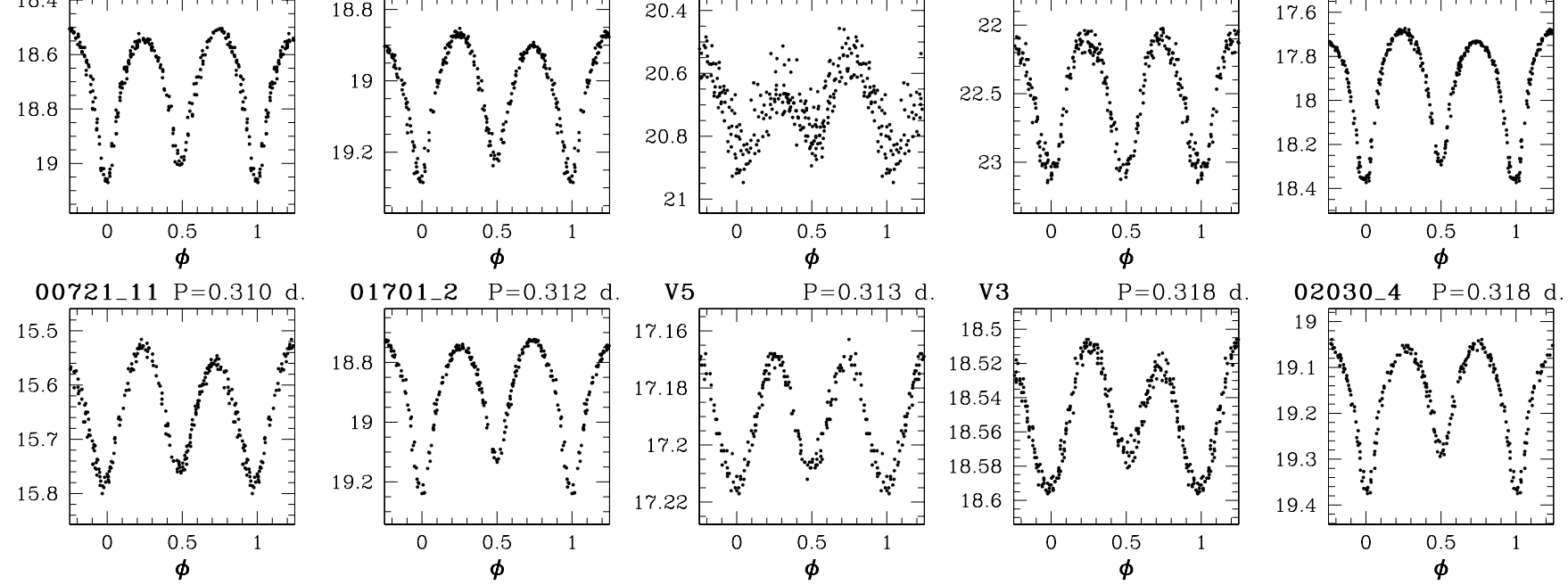

$017012 \quad P=0.312 d$

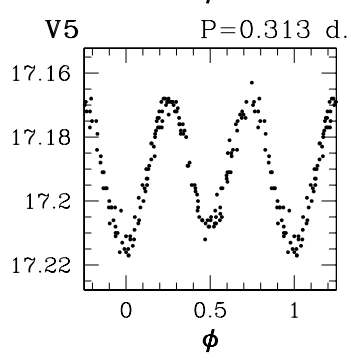

V3

$\mathrm{P}=0.318 \mathrm{~d}$.

02030_4 $P=0.318 d$.
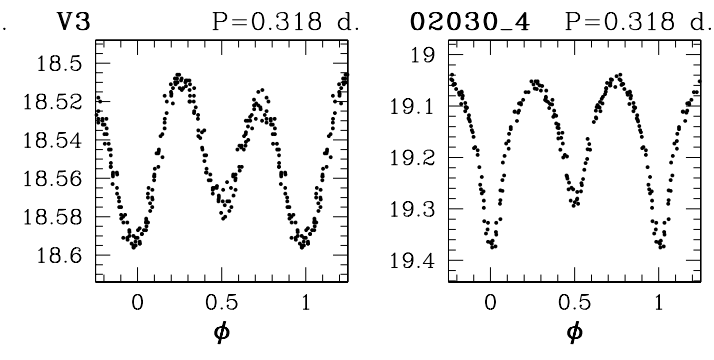

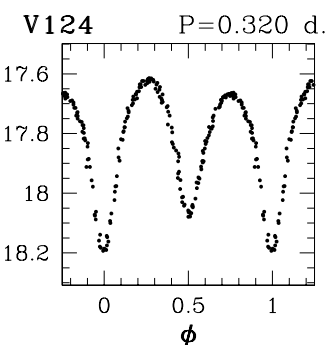

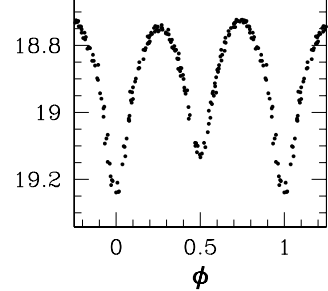

V4

V27

22.4 मे से
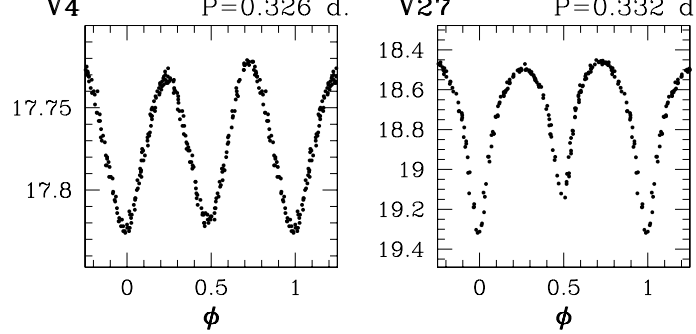

V8

$P=0.334 \mathrm{~d}$

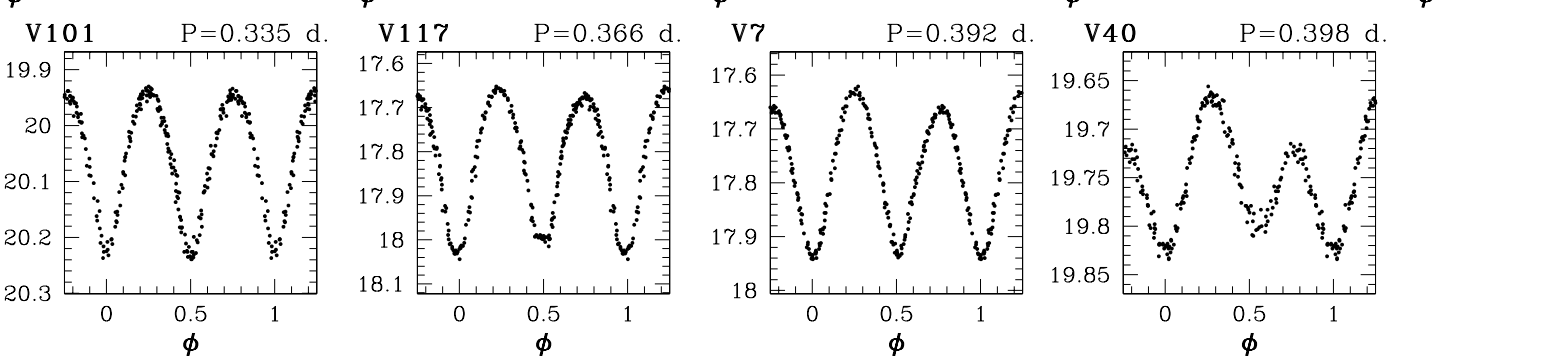

Fig. A.1. Contact variables. 
F. De Marchi et al.: Variable stars in the open cluster NGC 6791 and its surrounding field, Online Material p 5

Table A.3. Eclipsing variables. $V$ is the brightness at maximum and $T_{0}$ is the time of the primary minimum.

\begin{tabular}{|c|c|c|c|c|c|c|c|c|c|c|c|c|c|}
\hline Star & Type & $\alpha_{2000}$ & $\delta_{2000}$ & $\begin{array}{c}V \\
{[\mathrm{mag}]}\end{array}$ & $\begin{array}{c}\langle B-V\rangle \\
{[\mathrm{mag}]}\end{array}$ & $\begin{array}{c}\langle V-I\rangle \\
{[\mathrm{mag}]}\end{array}$ & Ref. & $\begin{array}{l}T_{0} \\
{[\mathrm{HJD}-2452400]}\end{array}$ & $\begin{array}{l}\text { Period } \\
\text { [d] }\end{array}$ & $\begin{array}{l}\text { Ampl. } \\
{[\mathrm{mag}]}\end{array}$ & Memb. & $\begin{array}{l}\text { Distance } \\
\text { [arcmin] }\end{array}$ & Notes \\
\hline V119 & EB & 19.351961 & 37.916328 & 18.15 & 1.13 & 1.33 & $\mathrm{k}$ & 59.879 & 0.30197 & 0.15 & $\mathrm{~m} ?$ & 9.1 & \\
\hline B4 & $\mathrm{E}:$ & 19.353589 & 37.764290 & 17.87 & -0.13 & -0.15 & $\mathrm{k}$ & 54.788 & 0.39841 & 0.02 & 40 & 4.0 & \\
\hline V29 & EB & 19.354796 & 37.751386 & 20.00 & 1.23 & 1.61 & $\mathrm{k}$ & 69.012 & 0.43662 & 0.22 & $\mathrm{~m}$ & 4.9 & $\begin{array}{l}\text { Light curve distortion } \\
\text { at maximum light }\end{array}$ \\
\hline 01558_5 & EB & 19.372697 & 37.953469 & 19.15 & & & & 69.028 & 0.52910 & 0.28 & nd2 & 20.6 & \\
\hline 01393_1 & EA & 19.329588 & 37.970392 & 21.23 & & & & 59.326 & 0.58998 & 0.56 & nd2 & 17.7 & \\
\hline 00331_3 & $\mathrm{EB}$ & 19.352367 & 37.864391 & 19.73 & 1.20 & 1.36 & $\mathrm{k}$ & 68.815 & 0.7347 & 0.13 & $\mathrm{~m} ?$ & 6.4 & \\
\hline V11 & EA & 19.342575 & 37.804802 & 19.38 & 0.96 & 1.22 & $\mathrm{k}$ & 67.875 & 0.8833 & 0.48 & $\mathrm{~m} ?$ & 4.4 & \\
\hline 05736_9 & EA & 19.348484 & 37.721855 & 20.20 & 1.21 & & $\mathrm{k}$ & 68.333 & 1.210 & 0.29 & $\mathrm{~m}$ & 3.0 & \\
\hline V12 & EB & 19.345259 & 37.849083 & 17.52 & 0.96 & & $\mathrm{k}$ & 64.103 & 1.524 & 0.06 & 96 & 5.1 & \\
\hline 00645_10 & EA & 19.354692 & 37.710104 & 20.60 & 1.33 & 1.46 & $\mathrm{k}$ & 60.893 & 1.451 & 0.20 & $\mathrm{~m}$ & 6.0 & \\
\hline V9 & EB & 19.346634 & 37.777035 & 17.15 & 1.23 & 1.38 & $\mathrm{k}$ & 63.873 & 3.2 & 0.20 & 82 & 1.1 & \\
\hline V107 & EA & 19.355068 & 37.761553 & 17.97 & 0.93 & 1.00 & $\mathrm{k}$ & 64.433 & 3.27 & 0.24 & $\mathrm{~m}$ ? & 5.0 & $\begin{array}{l}\text { Minima at the very } \\
\text { beginning of the night. }\end{array}$ \\
\hline V109 & EA & 19.342716 & 37.793961 & 20.73 & 1.46 & 1.60 & $\mathrm{k}$ & 69.021 & 3.70 & 0.86 & $\mathrm{~m} ?$ & 4.0 & \\
\hline 00219_11 & EA & 19.364048 & 37.827507 & 17.94 & 0.92 & 0.87 & $\mathrm{k}$ & 67.913 & & 0.52 & $\mathrm{~nm}$ & 11.9 & \\
\hline $00663 \_4$ & EA & 19.359652 & 37.894062 & 17.49 & 0.79 & 0.83 & $\mathrm{k}$ & 65.003 & & 0.49 & $\mathrm{~nm}$ & 11.0 & \\
\hline 00671_2 & EA & 19.336983 & 37.903919 & 18.46 & 0.70 & 0.91 & $\mathrm{k}$ & 64.968 & & 0.07 & $\mathrm{~nm}$ & 11.2 & \\
\hline 00828_5 & EA & 19.367917 & 37.885330 & 21.28 & & & & 64.979 & & 0.38 & nd2 & 15.7 & \\
\hline 00938_2 & EA & 19.338285 & 37.861633 & 20.57 & 1.20 & 1.80 & $\mathrm{k}$ & 67.867 & & 0.60 & $\mathrm{~nm}$ & 8.8 & \\
\hline 00997_10 & EA & 19.355692 & 37.799655 & 19.58 & 0.76 & 0.83 & $\mathrm{k}$ & 68.020 & & 0.20 & $\mathrm{~nm}$ & 5.7 & \\
\hline 01709_1 & EA & 19.330890 & 37.932268 & 18.04 & & & & 64.818 & & 0.32 & nd2 & 15.5 & \\
\hline 01731_10 & EA & 19.357813 & 37.672819 & 19.27 & 0.67 & 0.80 & $\mathrm{k}$ & 64.783 & & 0.34 & $\mathrm{~nm}$ & 9.1 & $\begin{array}{l}\text { Minimum at the very } \\
\text { beginning of the night. }\end{array}$ \\
\hline 01780_8 & EA & 19.340677 & 37.655354 & 22.19 & & 2.25 & $\mathrm{k}$ & 68.969 & & 0.66 & $\mathrm{~nm}$ & 8.7 & \\
\hline 02461_8 & EA & 19.342779 & 37.741798 & 19.34 & 1.06 & 1.15 & $\mathrm{k}$ & 60.968 & & 0.11 & 88 & 4.2 & \\
\hline 01740_7 & EA & 19.330623 & 37.638774 & 21.30 & & & & 69.007 & & 0.33 & $\mathrm{nd} 2$ & 14.8 & $\begin{array}{l}\text { Maybe another minimum } \\
\text { at } 64.40\end{array}$ \\
\hline 02045_12 & EA & 19.381420 & 37.712211 & 17.16 & & & & 68.030 & & 0.26 & nd2 & 24.0 & Other minimum at 63.39 \\
\hline 02241_11 & EA & 19.371472 & 37.827819 & 19.21 & & & & 68.823 & & 0.53 & $\mathrm{nd} 2$ & 17.0 & $\begin{array}{l}\text { Maybe another minimum } \\
\text { at } 63.50\end{array}$ \\
\hline $00346 \_5$ & EA & 19.365356 & 37.929481 & 20.16 & & & & 65.014 & & 0.17 & $\mathrm{nd} 2$ & 15.5 & $\begin{array}{l}\text { Other minimum at } \\
\text { at } 68.410 . \text { Short in time. }\end{array}$ \\
\hline 00631_12 & EA & 19.375473 & 37.649140 & 17.92 & & & & 68.753 & & 0.30 & $\mathrm{nd} 2$ & 20.9 & $\begin{array}{l}\text { Minimum at the very } \\
\text { beginning of the night. }\end{array}$ \\
\hline 01511_10 & EA & 19.357145 & 37.689907 & 19.74 & 1.45 & 1.86 & $\mathrm{k}$ & & & 0.50 & $\mathrm{~nm}$ & 8.1 & Two Minima at night extrema. \\
\hline V60 & EA & 19.350189 & 37.762493 & 18.68 & 0.96 & & $\mathrm{k}$ & 67.951 & & 0.39 & 91 & 1.6 & \\
\hline V80 & EA & 19.351799 & 37.791061 & 17.90 & 0.94 & & $\mathrm{k}$ & 67.607 & 4.631 & 0.10 & 86 & 2.9 & Shallow eclipse? \\
\hline V108 & EA & 19.352606 & 37.823467 & 21.15 & 1.27 & 1.90 & $\mathrm{k}$ & 69.080 & & 0.86 & $\mathrm{~nm}$ & 4.5 & \\
\hline
\end{tabular}


F. De Marchi et al.: Variable stars in the open cluster NGC 6791 and its surrounding field, Online Material p 6

Table A.4. Rotational variables with a single-wave light curve. $V$ is the mean brightness value. $T_{0}$ indicates the time of the maximum brightness.

\begin{tabular}{|c|c|c|c|c|c|c|c|c|c|c|c|}
\hline$\overline{\text { Star }}$ & 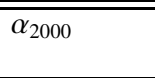 & " $\delta_{2000}$ & $\begin{array}{c}V \\
{[\mathrm{mag}]}\end{array}$ & $\begin{array}{c}\langle B-V\rangle \\
{[\mathrm{mag}]}\end{array}$ & $\begin{array}{l}\bar{V}-I\rangle \\
{[\mathrm{mag}]}\end{array}$ & Ref. & $\begin{array}{l}T_{0} \\
\text { [HJD-2 452 400] }\end{array}$ & $\begin{array}{l}\text { Period } \\
\text { [d] }\end{array}$ & $\begin{array}{l}\text { Ampl. } \\
\text { [mag] }\end{array}$ & "Memb. & $\begin{array}{l}\text { Distance } \\
\text { [arcmin] }\end{array}$ \\
\hline $02268 \_12$ & 19.382495 & 37.798839 & 17.42 & & & & 60.323 & 0.30910 & 0.02 & nd2 & 24.6 \\
\hline 02418_3 & 19.349085 & 38.016541 & 21.68 & & & & 59.901 & 0.36498 & 0.12 & nd2 & 14.7 \\
\hline $00513 \_2$ & 19.336012 & 37.940765 & 20.12 & 1.18 & 1.51 & $\mathrm{k}$ & 60.786 & 0.45272 & 0.08 & $\mathrm{~m} ?$ & 13.3 \\
\hline 02292_10 & 19.359535 & 37.710207 & 16.24 & 1.11 & 1.25 & $\mathrm{k}$ & 61.298 & 0.46371 & 0.06 & $\mathrm{~nm}$ & 9.0 \\
\hline 01497_11 & 19.368601 & 37.770512 & 18.58 & & & & 61.737 & 0.56508 & 0.01 & nd2 & 14.6 \\
\hline 01776_4 & 19.354509 & 37.935883 & 19.70 & 0.99 & 1.16 & $\mathrm{k}$ & 61.154 & 0.68029 & 0.06 & $\mathrm{~nm}$ & 10.9 \\
\hline 00301_5 & 19.365041 & 37.906284 & 22.10 & & & & 62.833 & 0.70170 & 0.21 & nd2 & 14.5 \\
\hline 00088_8 & 19.333437 & 37.744888 & 21.92 & & & & 65.464 & 0.70594 & 0.11 & nd2 & 10.5 \\
\hline V43 & 19.344337 & 37.641777 & 19.58 & 1.62 & 2.97 & $\mathrm{k}$ & 55.976 & 0.75759 & 0.08 & $\mathrm{~nm}$ & 8.2 \\
\hline $00554 \_8$ & 19.335732 & 37.664570 & 21.32 & 1.22 & 1.48 & $\mathrm{k}$ & 65.543 & 0.821 & 0.13 & $\mathrm{~nm}$ & 10.9 \\
\hline 00612_10 & 19.354604 & 37.729215 & 22.97 & 0.79 & 2.42 & $\mathrm{~s}$ & 68.050 & 0.91581 & 0.24 & $\mathrm{~m} ?$ & 5.3 \\
\hline 01105_12 & 19.377359 & 37.737573 & 19.31 & & & & 62.539 & 0.91746 & 0.04 & nd2 & 21.0 \\
\hline $00913 \_5$ & 19.368442 & 37.859339 & 20.89 & & & & 62.405 & 1.06386 & 0.24 & $\mathrm{nd} 2$ & 15.4 \\
\hline 04803_9 & 19.347698 & 37.796043 & 21.81 & 1.33 & 1.84 & $\mathrm{~s}$ & 61.799 & 1.1034 & 0.17 & m (B06) & 1.5 \\
\hline 01606_11 & 19.368978 & 37.736585 & 20.36 & & & & 62.390 & 1.12360 & 0.10 & nd2 & 15.0 \\
\hline V82 & 19.344366 & 37.793381 & 19.01 & 1.00 & 1.02 & $\mathrm{k}$ & 56.481 & 1.1568 & 0.04 & $\mathrm{~m}$ & 2.9 \\
\hline V34 & 19.335878 & 37.736183 & 19.30 & 1.16 & 1.41 & $\mathrm{k}$ & 54.955 & 1.20486 & 0.18 & $\mathrm{~nm}$ & 8.9 \\
\hline 05302_3 & 19.344669 & 37.968491 & 18.90 & & & & 63.224 & 1.3334 & 0.15 & nd2 & 12.1 \\
\hline 06553_9 & 19.349134 & 37.672577 & 19.26 & 1.04 & 1.13 & $\mathrm{~s}$ & 61.421 & 1.3485 & 0.08 & $\mathrm{~m}$ & 6.0 \\
\hline 00471_12 & 19.374883 & 37.674950 & 19.35 & & & & 61.900 & 1.3513 & 0.06 & nd2 & 20.0 \\
\hline 00110_5 & 19.363887 & 38.001389 & 21.69 & & & & 62.103 & 1.4085 & 0.08 & nd2 & 17.8 \\
\hline 01874_2 & 19.342730 & 37.912987 & 21.96 & & & & 64.944 & 1.5503 & 0.14 & nd1 & 9.3 \\
\hline V111 & 19.346970 & 37.812141 & 20.67 & 1.46 & 1.66 & $\mathrm{~s}$ & 61.673 & 1.5626 & 0.15 & $\mathrm{~nm}$ & 2.5 \\
\hline V37 & 19.355072 & 37.852001 & 19.58 & 1.65 & 2.48 & $\mathrm{k}$ & 55.721 & 1.6130 & 0.06 & $\mathrm{~nm}$ & 6.9 \\
\hline 01724_9 & 19.344957 & 37.785362 & 20.73 & 1.29 & 1.69 & $\mathrm{~s}$ & 64.410 & 1.6130 & 0.17 & $\mathrm{~m}$ & 2.4 \\
\hline 02285_10 & 19.359424 & 37.607155 & 19.26 & 1.34 & 1.57 & $\mathrm{k}$ & 63.495 & 1.6668 & 0.08 & $\mathrm{~nm}$ & 12.8 \\
\hline 01189_11 & 19.367513 & 37.809413 & 20.46 & & & & 64.500 & 1.7242 & 0.10 & nd2 & 14.0 \\
\hline 00016_5 & 19.363396 & 37.997513 & 18.68 & 1.54 & 2.52 & $\mathrm{k}$ & 63.157 & 1.7858 & 0.07 & $\mathrm{~nm}$ & 17.4 \\
\hline 00732_12 & 19.375889 & 37.717569 & 19.39 & & & & 61.992 & 1.852 & 0.04 & nd2 & 20.1 \\
\hline 00771_11 & 19.365949 & 37.766208 & 19.52 & & & & 62.492 & 1.852 & 0.09 & nd2 & 12.7 \\
\hline 00676_1 & 19.326459 & 37.929277 & 20.54 & & & & 65.481 & 1.887 & 0.11 & nd2 & 18.0 \\
\hline V38 & 19.351021 & 37.768288 & 18.82 & 0.96 & & $\mathrm{k}$ & 55.630 & 1.96 & 0.13 & 92 & 2.1 \\
\hline 02103_7 & 19.332028 & 37.696796 & 19.57 & 1.65 & & $\mathrm{k}$ & 60.891 & 2.041 & 0.05 & $\mathrm{~nm}$ & 12.3 \\
\hline 00575_12 & 19.375449 & 37.792459 & 19.63 & & & & 65.359 & 2.214 & 0.02 & nd2 & 19.5 \\
\hline 01914_1 & 19.331830 & 37.878855 & 17.71 & 0.73 & & $\mathrm{k}$ & 62.358 & 2.222 & 0.03 & $\mathrm{~nm}$ & 13.2 \\
\hline 03838_3 & 19.346848 & 37.964615 & 19.45 & 0.91 & 1.05 & $\mathrm{k}$ & 64.159 & 2.261 & 0.04 & $\mathrm{~nm}$ & 11.6 \\
\hline V44 & 19.326970 & 37.694771 & 18.38 & & & & 58.310 & 2.285 & 0.02 & nd2 & 15.7 \\
\hline 00321_1 & 19.324957 & 37.905996 & 19.49 & & & & 65.394 & 2.326 & 0.04 & nd2 & 18.3 \\
\hline 00810_5 & 19.367793 & 37.865903 & 18.17 & & & & 67.424 & 2.564 & 0.05 & nd2 & 15.1 \\
\hline 03079_9 & 19.346190 & 37.754753 & 19.23 & 1.14 & 1.26 & $\mathrm{k}$ & 66.630 & 2.640 & 0.07 & 93 & 1.7 \\
\hline 01821_12 & 19.380347 & 37.604033 & 20.13 & & & & 70.829 & 2.647 & 0.06 & nd2 & 25.1 \\
\hline 01309_11 & 19.367871 & 37.748052 & 20.81 & & & & 61.125 & 2.692 & 0.05 & nd2 & 14.2 \\
\hline 01956_12 & 19.380972 & 37.626492 & 21.39 & & & & 63.833 & 2.699 & 0.06 & nd2 & 25.0 \\
\hline 00815_1 & 19.327108 & 37.861623 & 19.75 & & & & 63.358 & 2.836 & 0.05 & $\mathrm{nd} 2$ & 15.8 \\
\hline 01659_8 & 19.340244 & 37.694355 & 21.27 & 1.51 & 1.85 & $\mathrm{k}$ & 64.446 & 2.840 & 0.17 & $\mathrm{~nm}$ & 7.2 \\
\hline 00293_11 & 19.364252 & 37.703417 & 15.82 & 0.80 & 0.88 & $\mathrm{k}$ & 63.606 & 2.941 & 0.05 & $\mathrm{~nm}$ & 12.2 \\
\hline 00215_5 & 19.364447 & 37.898928 & 22.26 & & & & 65.340 & 3.15 & 0.14 & nd2 & 13.9 \\
\hline 03209_10 & 19.362653 & 37.732028 & 18.61 & 1.54 & 2.57 & $\mathrm{k}$ & 66.016 & 3.17 & 0.06 & $\mathrm{~nm}$ & 10.7 \\
\hline 01313_1 & 19.329261 & 38.025661 & 21.27 & & & & 62.466 & 3.704 & 0.10 & nd2 & 20.3 \\
\hline 00277_8 & 19.334366 & 37.776566 & 19.52 & 1.54 & 2.68 & $\mathrm{k}$ & 60.691 & 4.167 & 0.05 & $\mathrm{~nm}$ & 9.7 \\
\hline 00179_5 & 19.364283 & 38.002234 & 17.18 & 0.91 & 0.90 & $\mathrm{k}$ & 64.764 & 4.323 & 0.04 & $\mathrm{~nm}$ & 18.0 \\
\hline 03039_10 & 19.362130 & 37.815850 & 16.58 & 0.79 & 0.87 & $\mathrm{k}$ & 65.634 & 4.423 & 0.02 & $\mathrm{~nm}$ & 10.4 \\
\hline 01555_4 & 19.355373 & 37.961205 & 21.22 & & & & 69.673 & 4.546 & 0.09 & nd2 & 12.5 \\
\hline 02614_11 & 19.372592 & 37.625244 & 17.22 & & & & 70.152 & 4.763 & 0.04 & nd2 & 19.6 \\
\hline 01149_2 & 19.339287 & 37.966995 & 17.80 & 0.90 & 0.93 & $\mathrm{k}$ & 67.803 & 5.0 & 0.04 & $\mathrm{~m} ?$ & 13.3 \\
\hline 02815_3 & 19.348427 & 37.967987 & 19.87 & 1.68 & 2.40 & $\mathrm{k}$ & 60.828 & 5.0 & 0.05 & $\mathrm{~nm}$ & 11.8 \\
\hline V46 & 19.355272 & 37.798869 & 18.65 & 1.18 & 1.36 & $\mathrm{k}$ & 56.886 & 5.2 & 0.14 & $\mathrm{~nm}$ & 5.4 \\
\hline 00357_5 & 19.365449 & 37.996214 & 20.91 & & & & 63.108 & 5.3 & 0.07 & nd2 & 18.3 \\
\hline 01484_7 & 19.329583 & 37.791249 & 20.30 & 1.63 & & $\mathrm{k}$ & 67.924 & 5.3 & 0.03 & $\mathrm{~nm}$ & 13.2 \\
\hline V14 & 19.347687 & 37.756874 & 18.58 & 0.93 & 1.05 & $\mathrm{k}$ & 55.933 & 5.45 & 0.05 & 0 & 0.9 \\
\hline V48 & 19.352076 & 37.718506 & 17.51 & 0.88 & & $\mathrm{k}$ & 65.223 & 5.65 & 0.09 & 96 & 4.3 \\
\hline
\end{tabular}


F. De Marchi et al.: Variable stars in the open cluster NGC 6791 and its surrounding field, Online Material p 7

Table A.4. continued: other rotational variables with a single-wave light curve. $V$ is the mean brightness value. $T_{0}$ indicates (one of) the times of maximum brightness.

\begin{tabular}{|c|c|c|c|c|c|c|c|c|c|c|c|}
\hline Star & $\alpha_{2000}$ & $\delta_{2000}$ & $\begin{array}{c}V \\
{[\mathrm{mag}]}\end{array}$ & $\begin{array}{c}\langle B-V\rangle \\
{[\mathrm{mag}]}\end{array}$ & $\begin{array}{c}\langle V-I\rangle \\
{[\mathrm{mag}]}\end{array}$ & Ref. & $\begin{array}{l}T_{0} \\
\text { [HJD-2 452 400] }\end{array}$ & $\begin{array}{l}\text { Period } \\
{[\mathrm{d}]}\end{array}$ & $\begin{array}{l}\text { Ampl. } \\
\text { [mag] }\end{array}$ & Memb. & $\begin{array}{l}\text { Distance } \\
\text { [arcmin] }\end{array}$ \\
\hline 00615_7 & 19.325908 & 37.753712 & 20.59 & & & & 59.885 & 5.882 & 0.14 & $\mathrm{nd} 2$ & 15.8 \\
\hline V89 & 19.349068 & 37.776783 & 18.75 & 0.88 & 1.20 & $\mathrm{~s}$ & 63.447 & 5.884 & 0.05 & $\mathrm{~nm}$ & 0.8 \\
\hline 00188_12 & 19.373720 & 37.632076 & 21.78 & & & & 67.253 & 6.25 & 0.06 & nd2 & 20.1 \\
\hline V17 & 19.344135 & 37.817928 & 17.92 & 1.20 & 1.28 & $\mathrm{k}$ & 63.211 & 6.523 & 0.04 & 88 & 3.9 \\
\hline 01122_4 & 19.357346 & 37.914520 & 18.84 & 1.03 & 1.12 & $\mathrm{k}$ & 65.843 & 6.69 & 0.11 & $\mathrm{~m}$ ? & 10.8 \\
\hline 03056_3 & 19.347979 & 37.869431 & 17.10 & 0.89 & 0.97 & $\mathrm{k}$ & 66.673 & 6.70 & 0.07 & $\mathrm{~nm}$ & 5.9 \\
\hline V51 & 19.353382 & 37.748795 & 19.94 & 1.22 & 1.21 & $\mathrm{k}$ & 63.624 & 6.72 & 0.09 & $\mathrm{~m}$ & 4.0 \\
\hline 00640_10 & 19.354585 & 37.604724 & 17.97 & 0.68 & 0.77 & $\mathrm{k}$ & 66.173 & 6.8 & 0.03 & $\mathrm{~nm}$ & 11.0 \\
\hline V52 & 19.355795 & 37.771935 & 17.49 & 0.88 & 0.88 & $\mathrm{k}$ & 64.345 & 7.06 & 0.03 & $\mathrm{~m}$ & 5.5 \\
\hline V53 & 19.350233 & 37.743187 & 18.72 & 0.89 & 0.93 & $\mathrm{k}$ & 69.294 & 7.47 & 0.04 & 86 & 2.3 \\
\hline 01431_10 & 19.357001 & 37.763810 & 17.72 & 1.33 & 1.45 & $\mathrm{k}$ & 67.953 & 7.64 & 0.26 & $\mathrm{~nm}$ & 6.4 \\
\hline 01616_11 & 19.369060 & 37.767818 & 17.26 & & & & 69.157 & 7.70 & 0.05 & nd2 & 14.9 \\
\hline 01478_3 & 19.350523 & 37.862484 & 17.15 & 1.03 & 1.11 & $\mathrm{k}$ & 72.123 & 7.96 & 0.07 & $\mathrm{~nm}$ & 5.7 \\
\hline 05877_9 & 19.348616 & 37.767616 & 20.22 & 1.32 & 1.49 & $\mathrm{k}$ & 64.272 & 8.0 & 0.12 & $\mathrm{~nm}$ & 0.5 \\
\hline
\end{tabular}

Table A.5. Rotational variables with a double-wave light curve. $V$ is the mean brightness value. $T_{0}$ indicates (one of) the times of maximum brightness.

\begin{tabular}{|c|c|c|c|c|c|c|c|c|c|c|c|}
\hline$\overline{\text { Star }}$ & 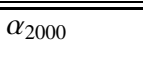 & 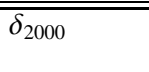 & $\begin{array}{c}V \\
{[\mathrm{mag}]}\end{array}$ & $\begin{array}{c}\langle B-V\rangle \\
{[\mathrm{mag}]}\end{array}$ & $\begin{array}{c}\langle V-I\rangle \\
{[\mathrm{mag}]}\end{array}$ & Ref. & $\begin{array}{l}T_{0} \\
\text { [HJD-2 452 400] }\end{array}$ & $\begin{array}{l}\text { Period } \\
\text { [d] }\end{array}$ & $\begin{array}{l}\text { Ampl. } \\
\text { [mag] }\end{array}$ & Memb. & $\begin{array}{l}\text { Distance } \\
\text { [arcmin] }\end{array}$ \\
\hline 004363 & 19.352205 & 37.878635 & 18.92 & 0.92 & 1.08 & $\mathrm{k}$ & 60.018 & 0.26601 & 0.04 & $\mathrm{~m}$ & 7.1 \\
\hline V2 & 19.354875 & 37.766689 & 19.74 & 0.93 & 1.21 & $\mathrm{k}$ & 59.712 & 0.27344 & 0.17 & $\mathrm{~nm}$ & 4.9 \\
\hline 02006_1 & 19.332258 & 38.043080 & 21.54 & & & & 60.448 & 0.37500 & 0.19 & $\mathrm{nd} 2$ & 19.8 \\
\hline 01175_5 & 19.370209 & 37.998342 & 19.73 & & & & 59.918 & 0.41481 & 0.09 & $\mathrm{nd} 2$ & 20.8 \\
\hline 00536_11 & 19.365067 & 37.716529 & 19.13 & & & & 60.460 & 0.43740 & 0.03 & nd2 & 12.6 \\
\hline 07483_9 & 19.349997 & 37.746311 & 21.28 & 1.32 & 1.70 & $\mathrm{~s}$ & 60.465 & 0.4375 & 0.17 & $\mathrm{~m}$ & 2.1 \\
\hline V41 & 19.347492 & 37.806892 & 19.09 & & & & 60.000 & 0.4798 & 0.07 & 77 & 2.2 \\
\hline V42 & 19.350058 & 37.714867 & 19.51 & 1.05 & 1.16 & $\mathrm{k}$ & 60.323 & 0.5068 & 0.10 & 92 & 3.7 \\
\hline $01362 \_7$ & 19.329000 & 37.662097 & 20.53 & & & & 60.502 & 0.5560 & 0.08 & nd2 & 15.1 \\
\hline 02270_11 & 19.371548 & 37.794338 & 17.95 & & & & 60.525 & 0.5679 & 0.05 & nd2 & 16.8 \\
\hline 01298_5 & 19.371044 & 38.002255 & 19.76 & & & & 60.402 & 0.5859 & 0.06 & $\mathrm{nd} 2$ & 21.4 \\
\hline V16 & 19.352108 & 37.802662 & 17.79 & 0.93 & 1.01 & $\mathrm{k}$ & 67.713 & 2.182 & 0.03 & 96 & 3.4 \\
\hline 00568_2 & 19.336329 & 37.881542 & 18.52 & & & & 60.007 & 2.704 & 0.08 & nd2 & 10.6 \\
\hline 00019_7 & 19.323382 & 37.710172 & 18.95 & & & & 65.473 & 5.882 & 0.12 & nd2 & 17.9 \\
\hline
\end{tabular}


F. De Marchi et al.: Variable stars in the open cluster NGC 6791 and its surrounding field, Online Material $p 8$
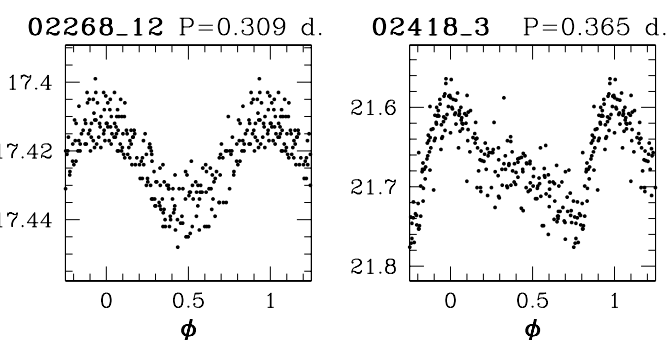

$00513 \_2 \quad \mathrm{P}=0.453 \mathrm{~d}$

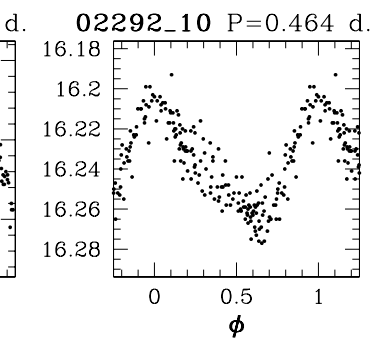

01497_11 P=0.565d

$017764 \quad P=0.680$
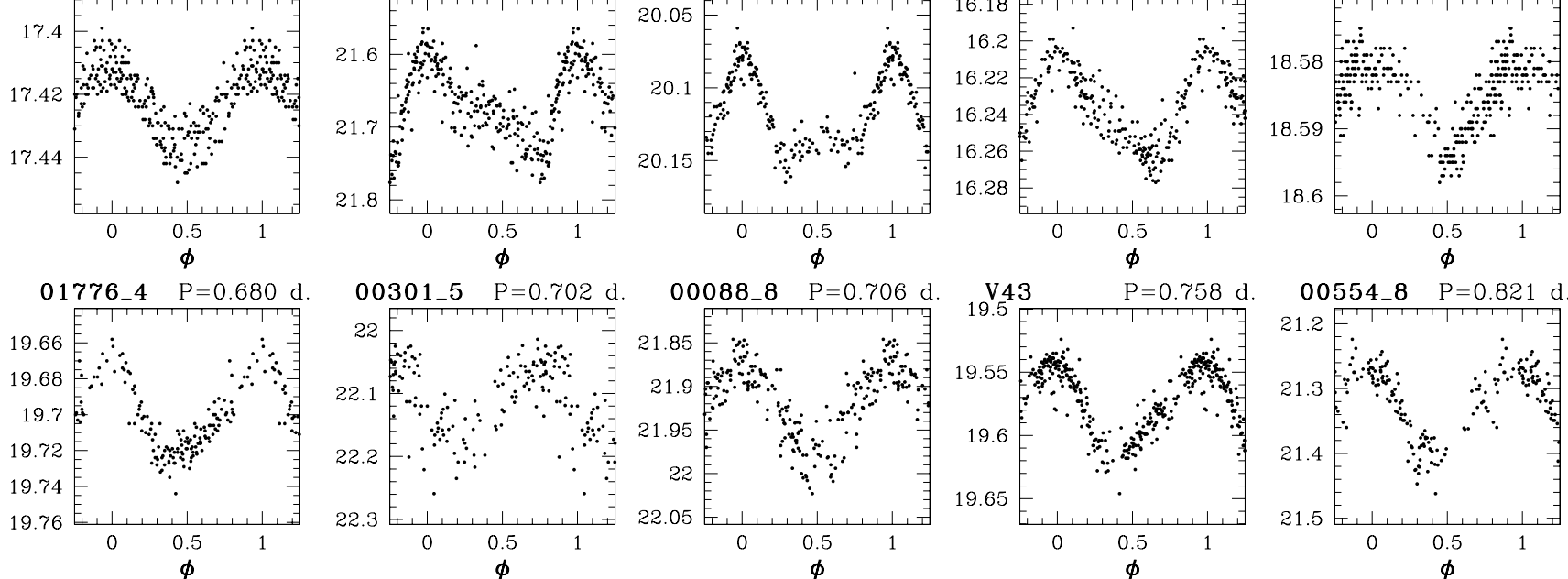

$000888 \quad \mathrm{P}=0.706 \mathrm{~d}$

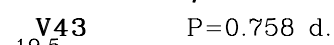

$005548 \quad \mathrm{P}=0.821 \mathrm{~d}$.
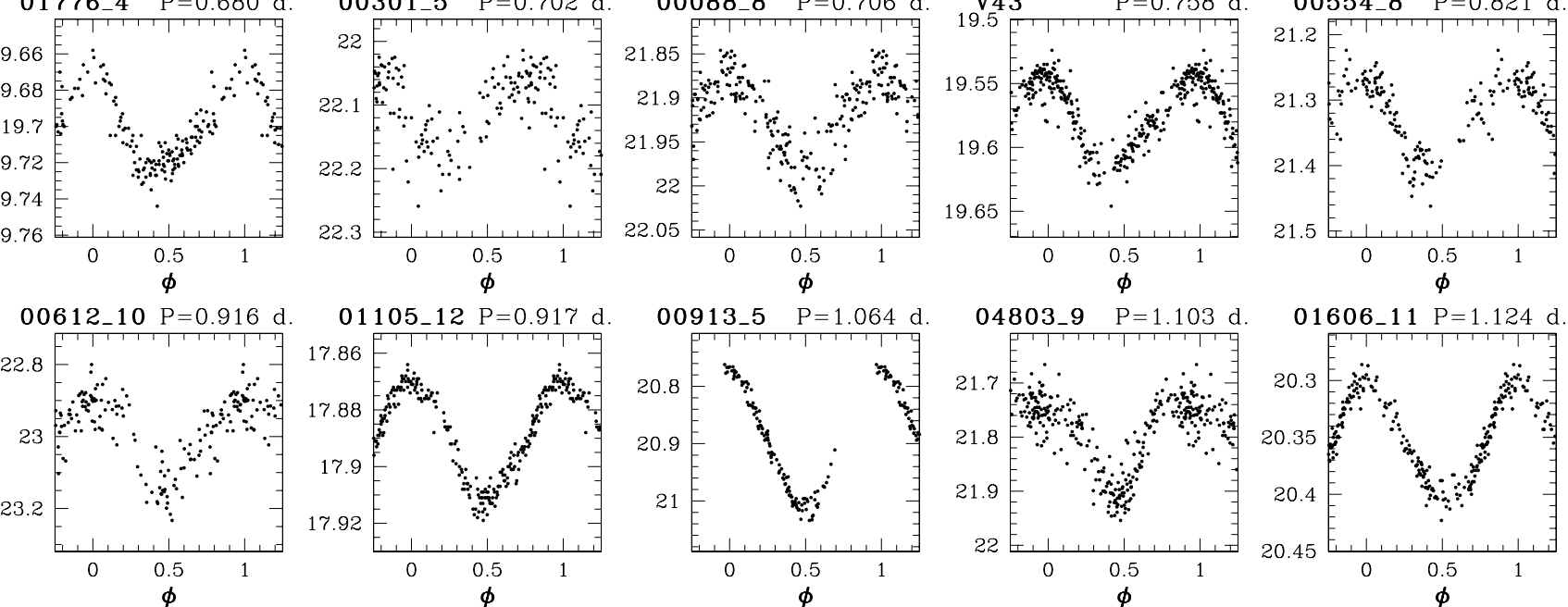

$01105-12 P=0.917 \mathrm{~d}$

$00913 \_\mathrm{P}=1.064 \mathrm{~d}$.

04803 -9 $P=1.103 d$.

01606_11 P=1.124d.
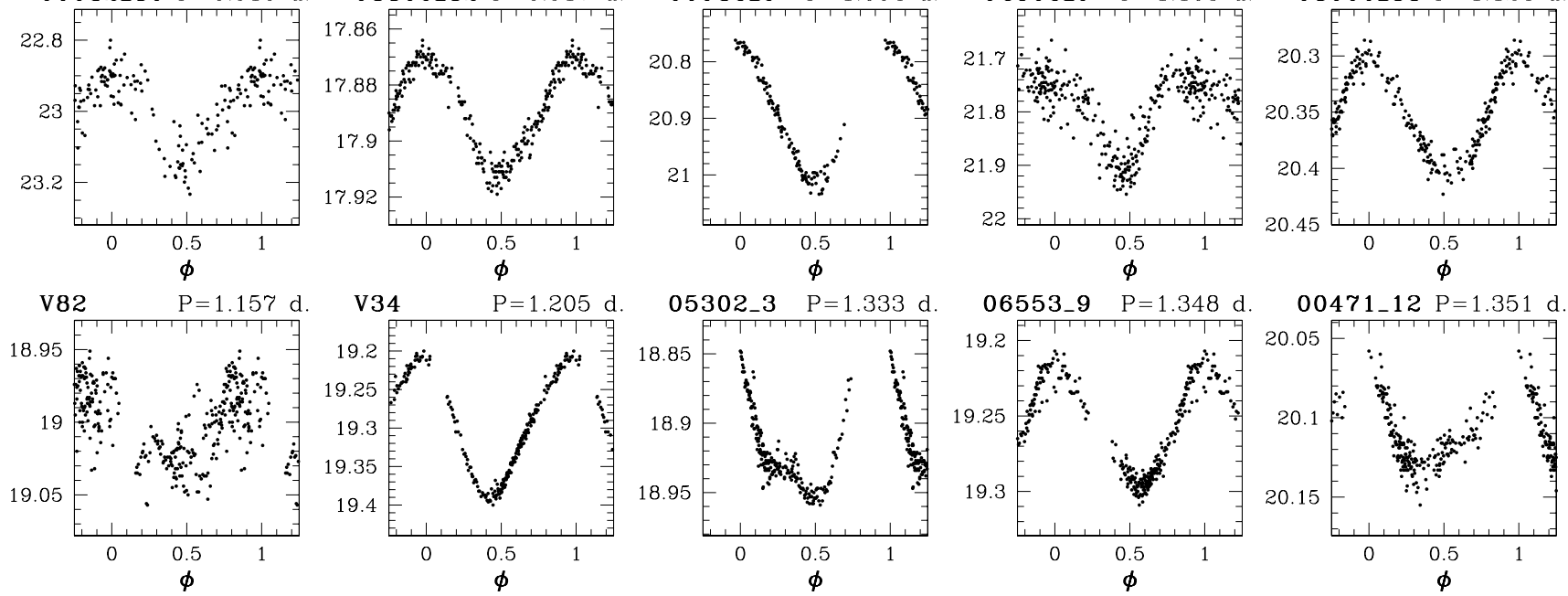

06553 9 $P=1.348 d$.
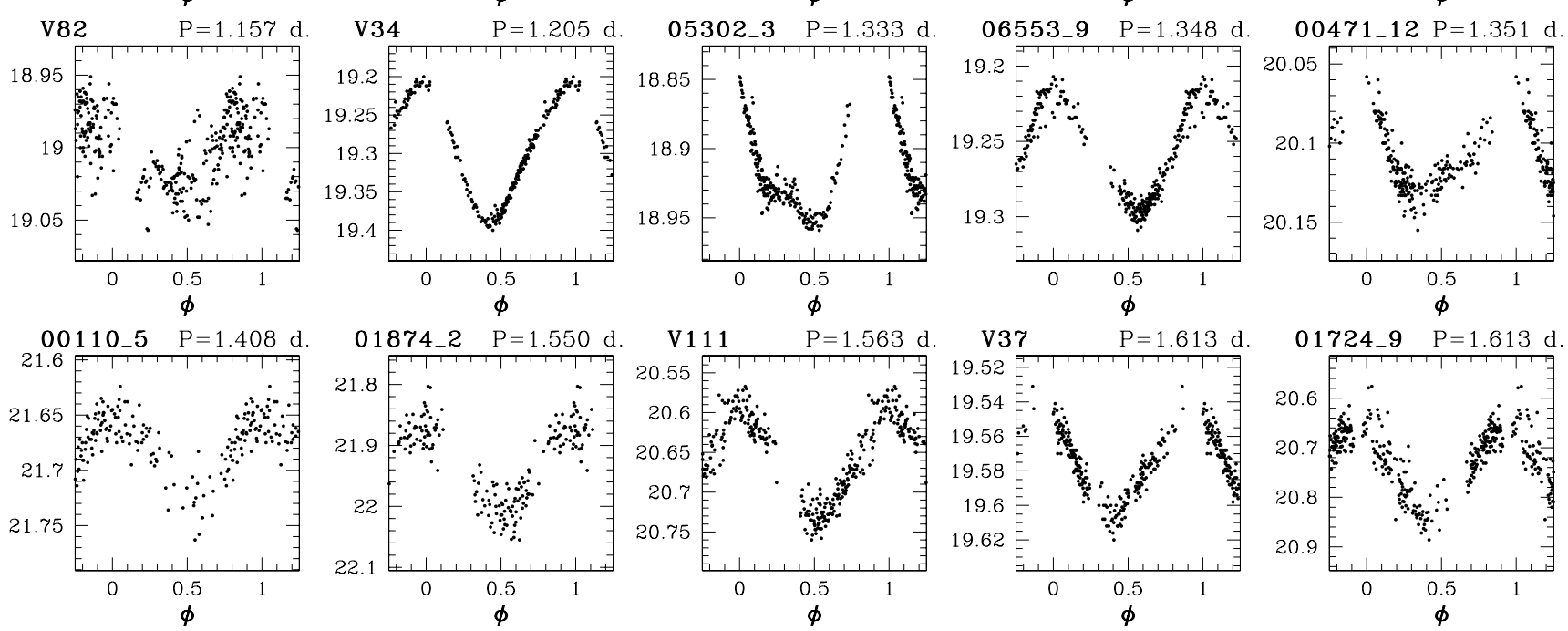

$01874 \_\mathrm{P}=1.550 \mathrm{~d}$.
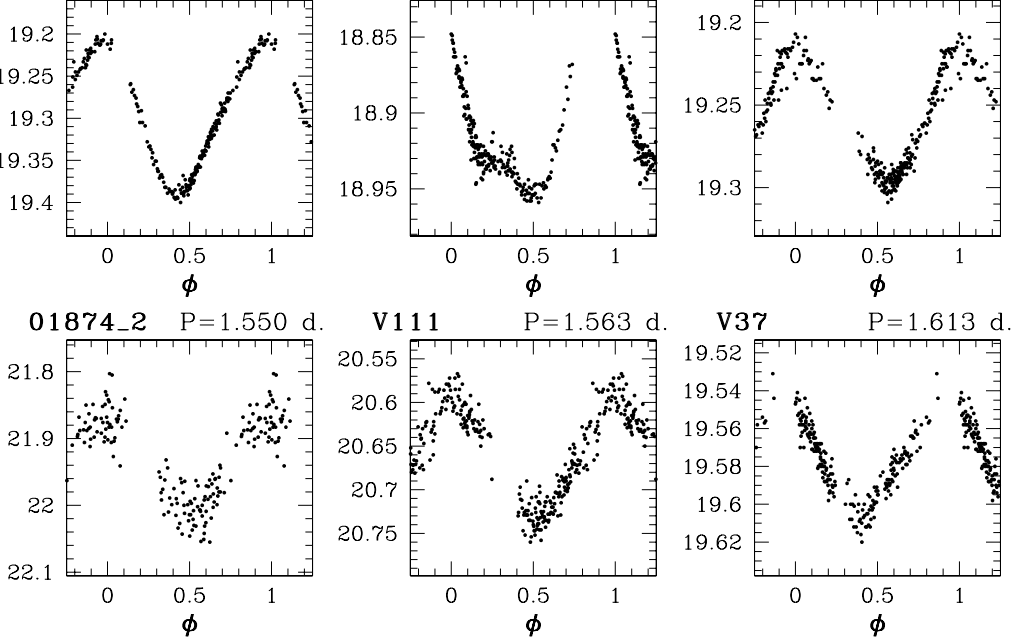

$01724 \_9 \quad \mathrm{P}=1.613 \mathrm{~d}$.

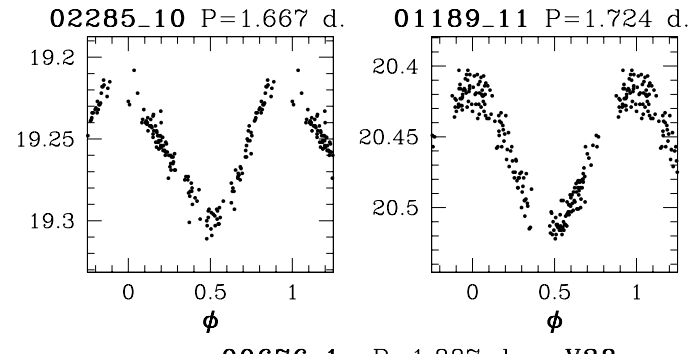

$0118911 \mathrm{P}=1.724 \mathrm{~d}$

$000165 \mathrm{P}=1.786 \mathrm{~d}$.

$0073212 \mathrm{P}=1.852 \mathrm{~d}$
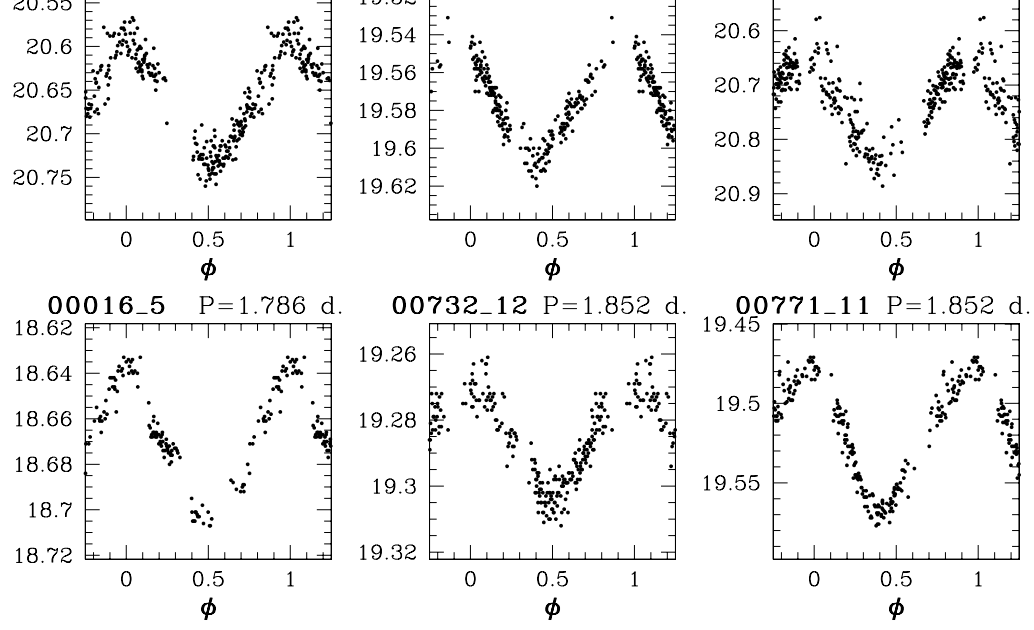

19.45771 $11 \mathrm{P}=1.852 \mathrm{~d}$.
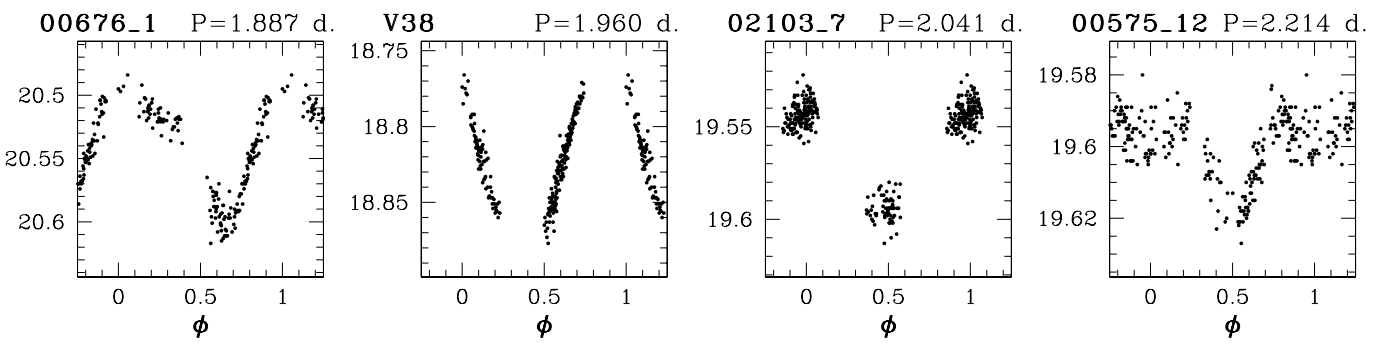

Fig. A.2. Rotational variables with a single-wave light curve, in some cases very distorted. 
F. De Marchi et al.: Variable stars in the open cluster NGC 6791 and its surrounding field, Online Material $p 9$
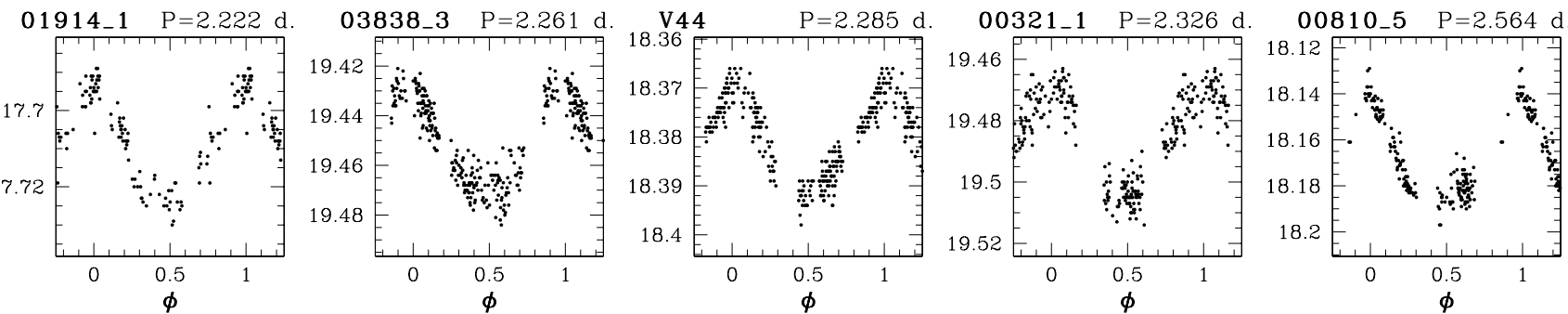

$030799 \quad \mathrm{P}=2.640 \mathrm{~d}$

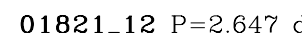

$0130911 \mathrm{P}=2.692$

$0195612 \mathrm{P}=2.699 \mathrm{~d}$

$008151 \quad P=2.836 \mathrm{~d}$
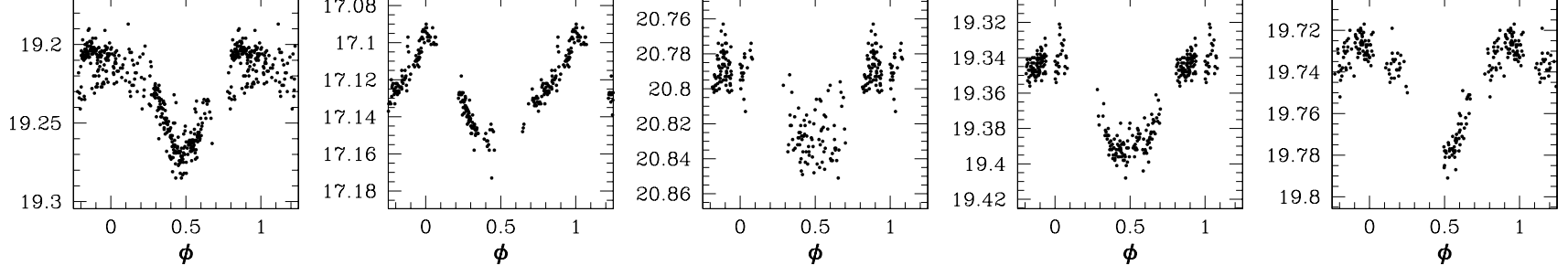

$016598 \quad \mathrm{P}=2.840 \mathrm{~d}$.

$00293-11 \mathrm{P}=2.941 \mathrm{~d}$

$00215-5 \quad P=3.150 \mathrm{~d}$

$03209+10 \mathrm{P}=3.170 \mathrm{~d}$

$01313-1 \quad \mathrm{P}=3.704 \mathrm{~d}$
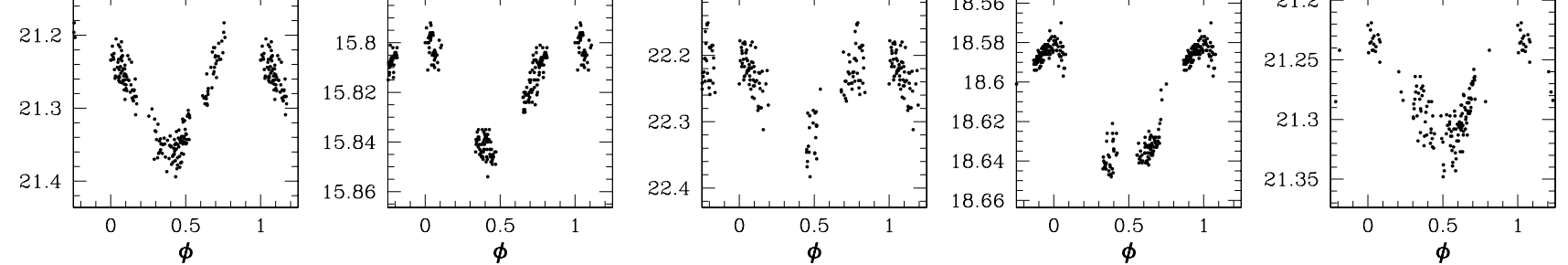

$002778 \quad \mathrm{P}=4.167 \mathrm{~d} . \quad 001795 \mathrm{P}=4.323 \mathrm{~d}$.

$03039+10 \quad P=4.423$

$01555 \quad 4 \quad \mathrm{P}=4.546 \mathrm{~d}$

$02614 \_11 \mathrm{P}=4.763 \mathrm{~d}$
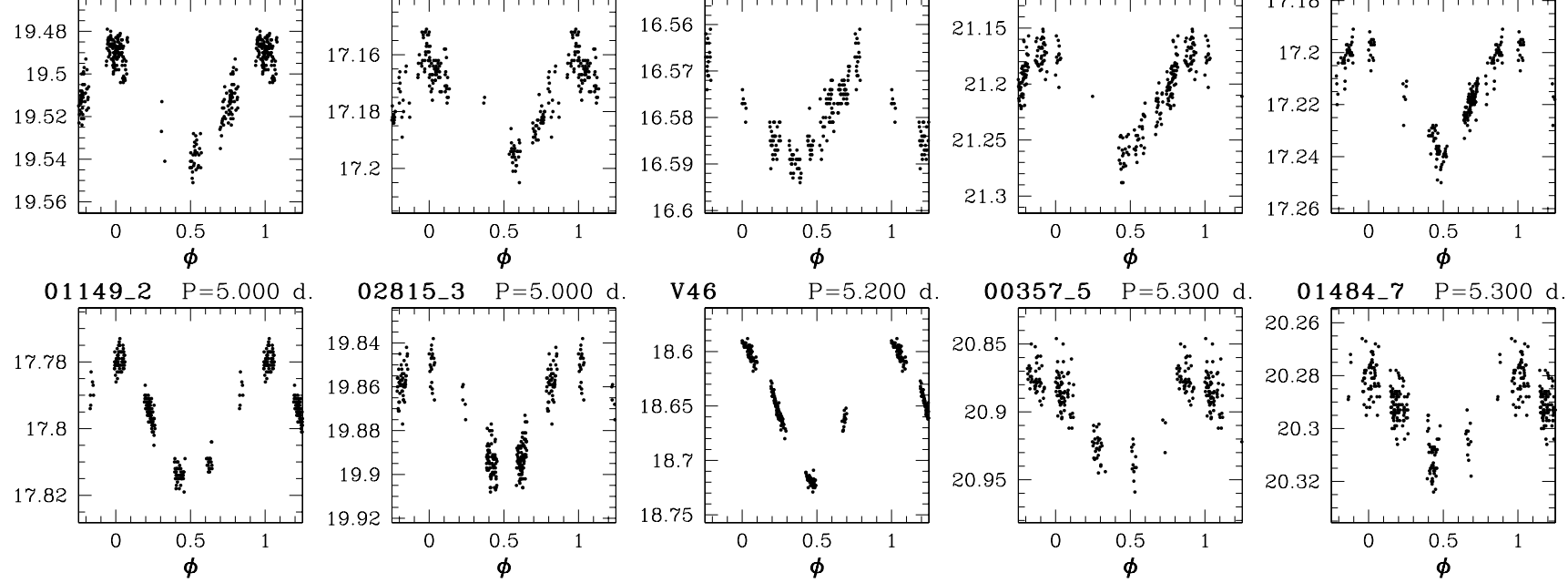

$00357-5$
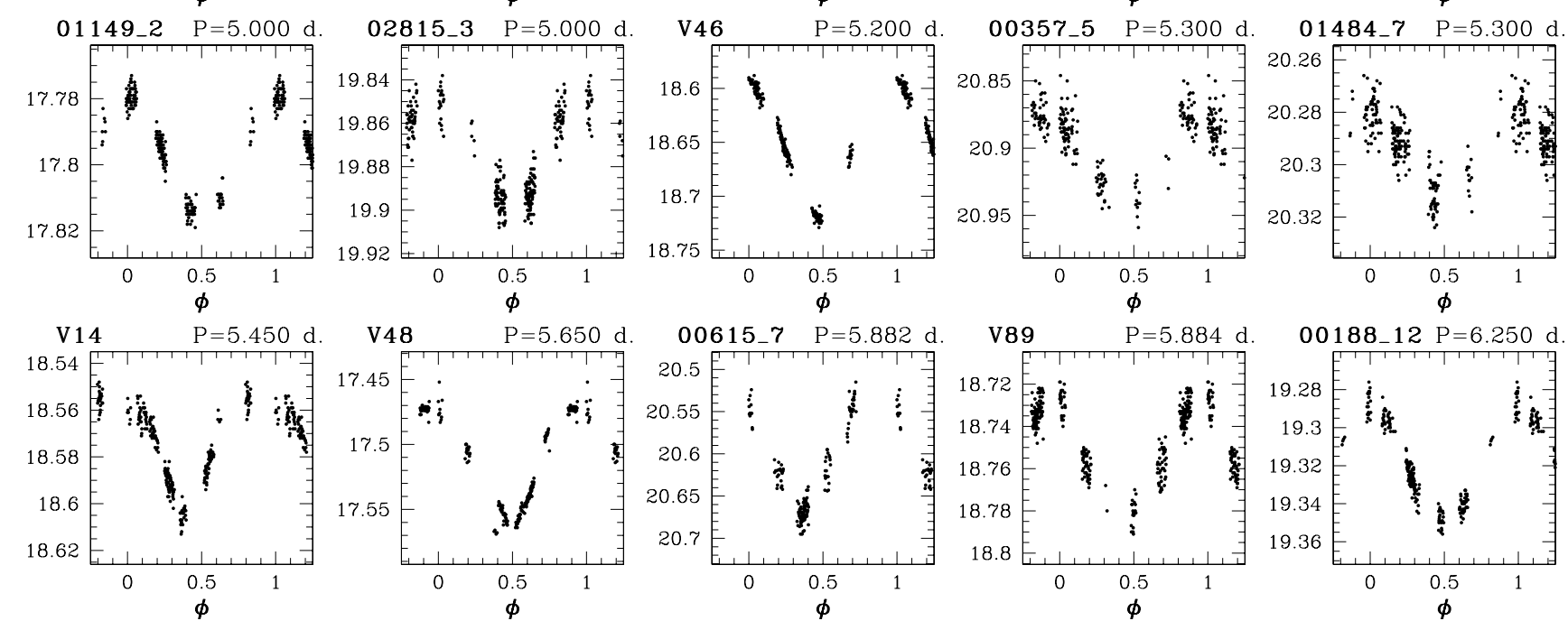

$006157 \quad \mathrm{P}=5.882 \mathrm{~d}$.
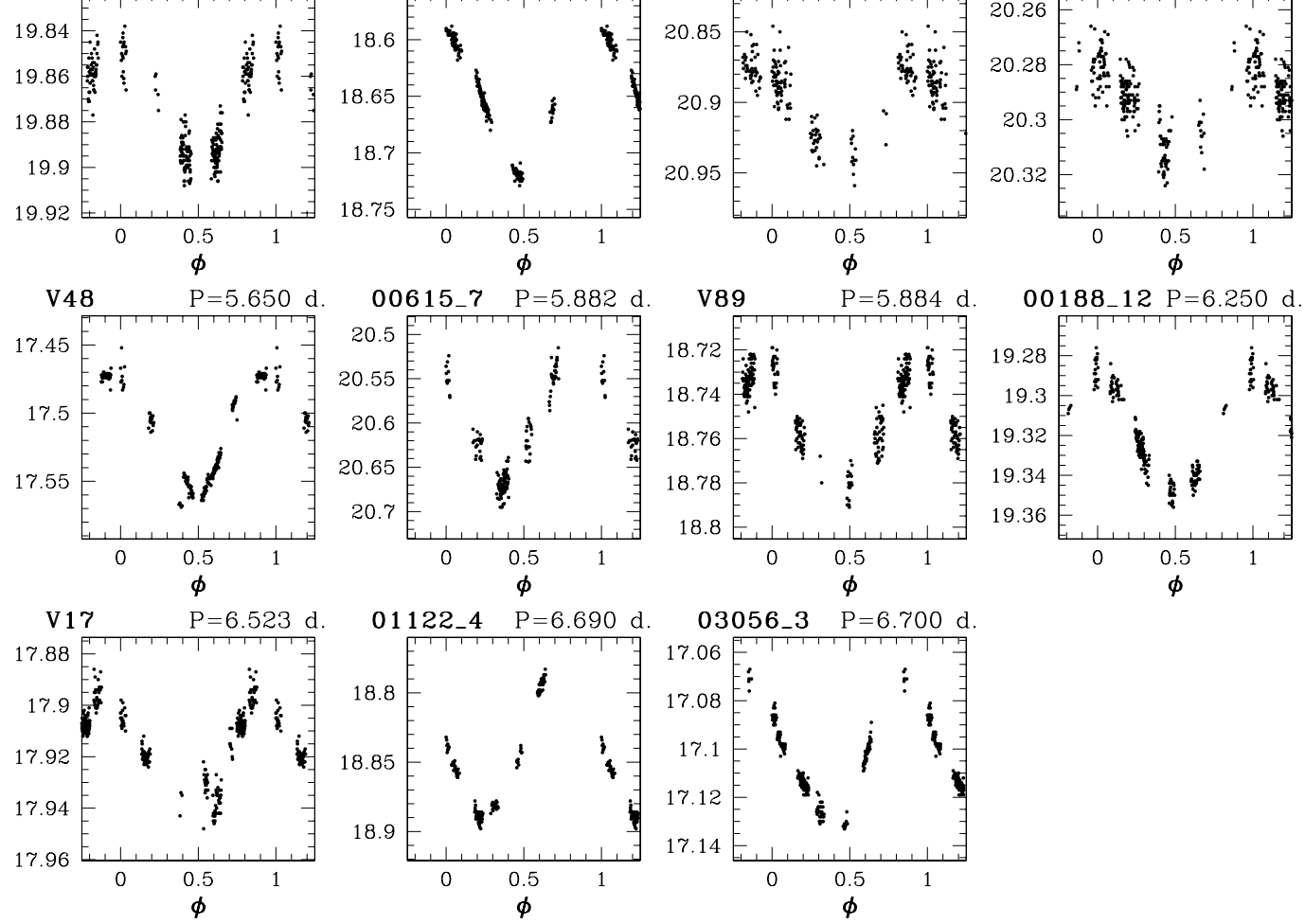

$0018812 \mathrm{P}=6.250 \mathrm{~d}$
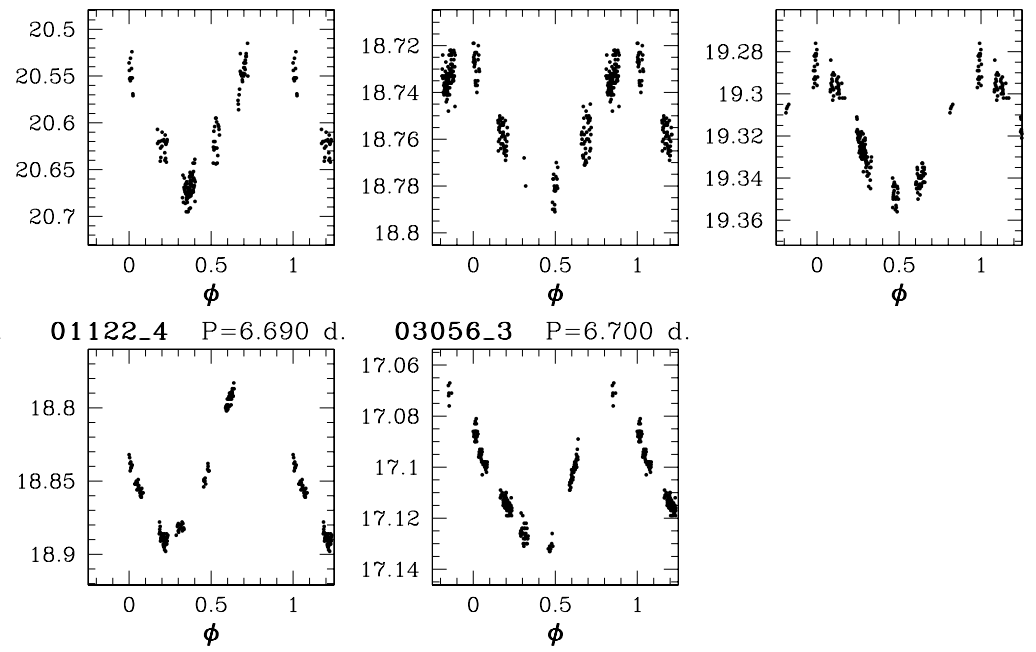

$03056 \_3 \quad \mathrm{P}=6.700 \mathrm{~d}$ 
F. De Marchi et al.: Variable stars in the open cluster NGC 6791 and its surrounding field, Online Material p 10

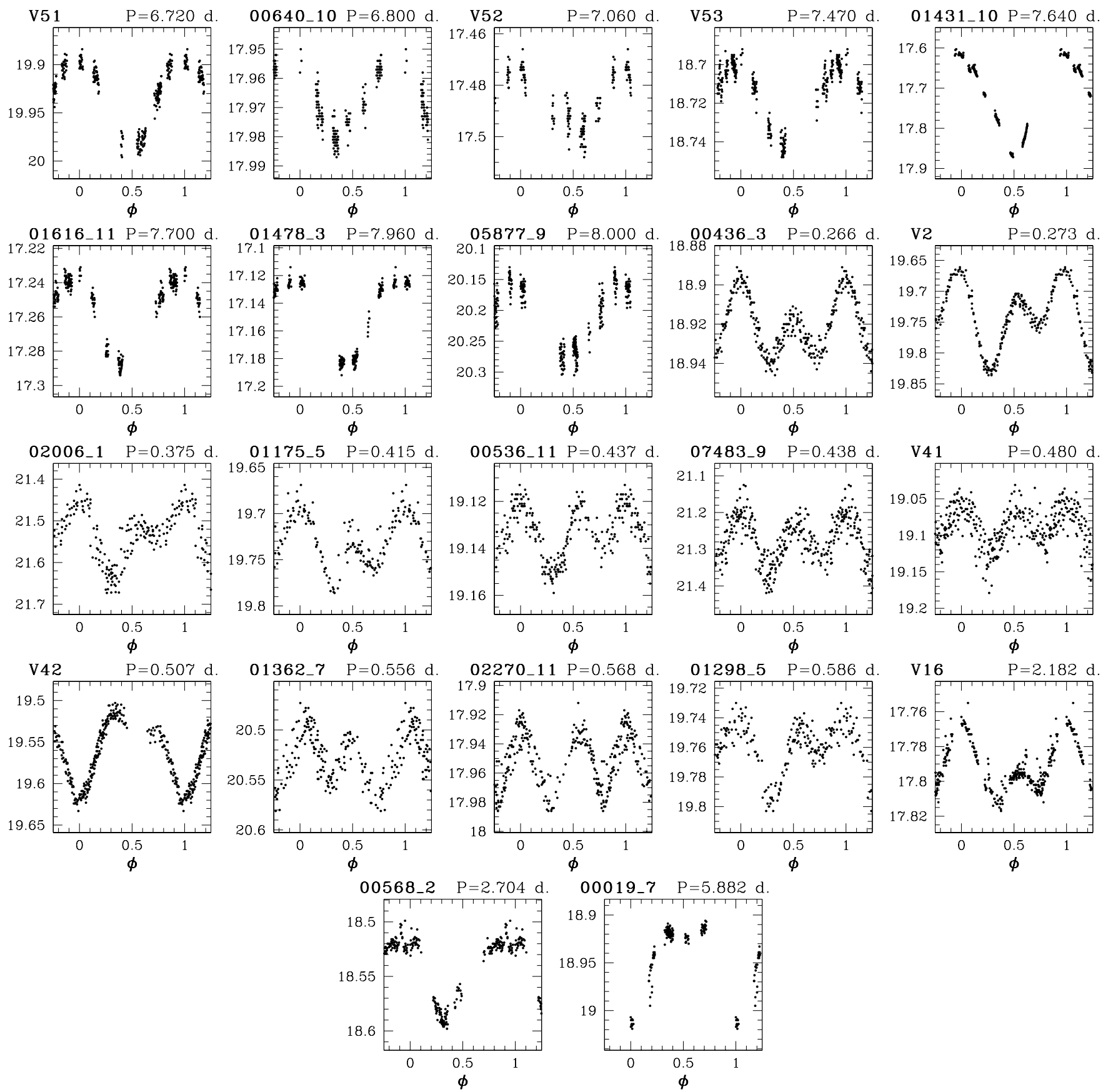

Fig. A.4. Rotational variables with a single-wave (from V51 to 05877_9) and a double-wave light curve. 
F. De Marchi et al.: Variable stars in the open cluster NGC 6791 and its surrounding field, Online Material p 11

Table A.6. Long period variable stars (ordered by increasing right ascension).

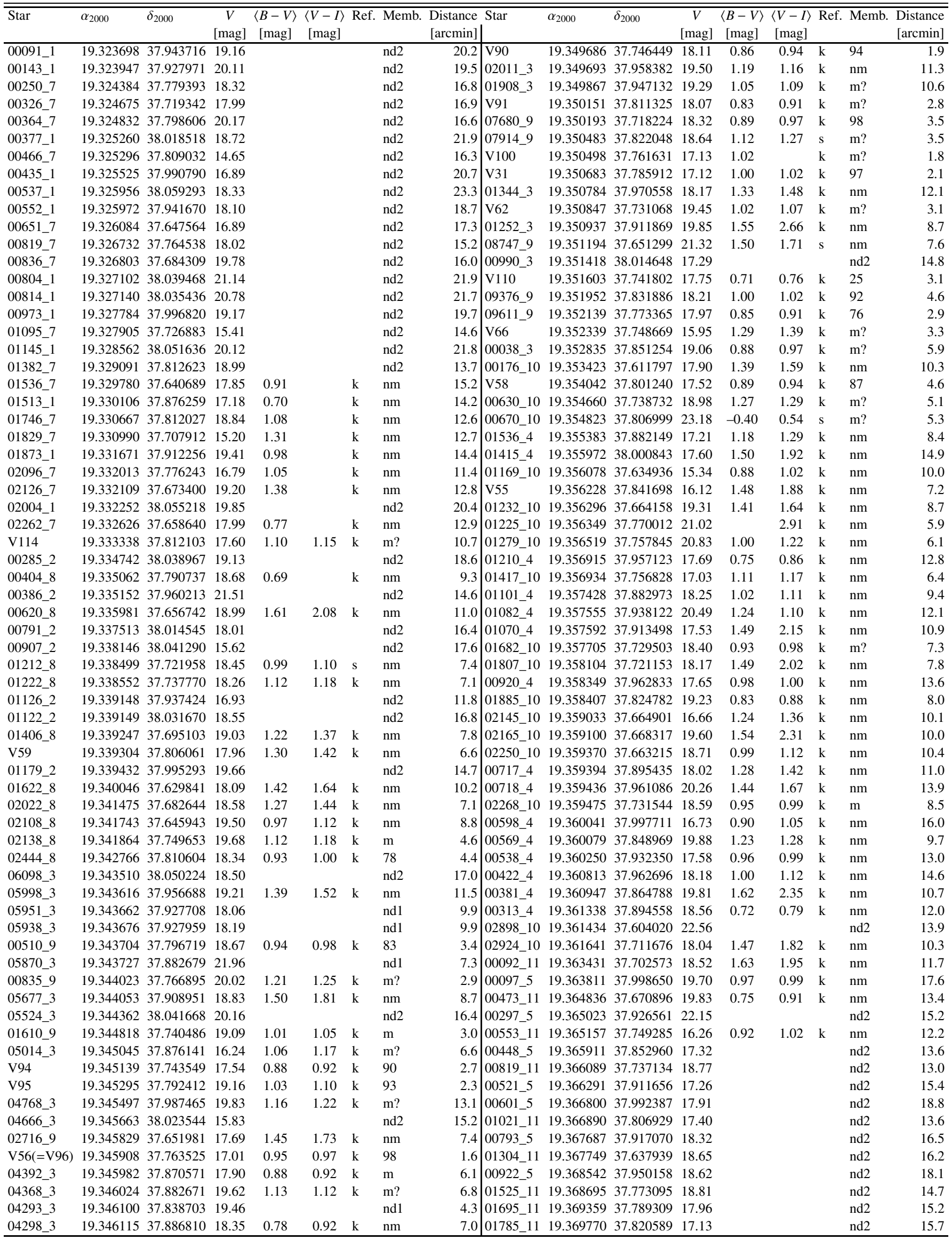


F. De Marchi et al.: Variable stars in the open cluster NGC 6791 and its surrounding field, Online Material p 12

Table A.6. continued.

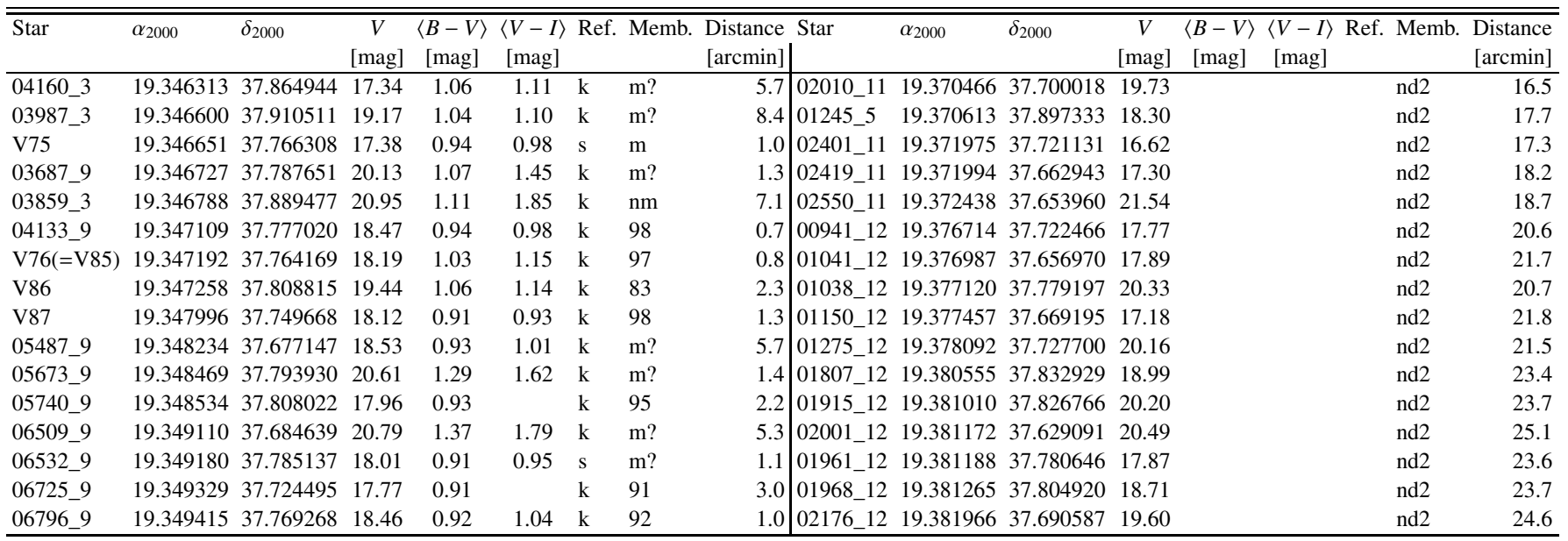

